\title{
Peripherally derived FGF21 promotes remyelination in the central nervous system
}

\author{
Mariko Kuroda, ${ }^{1}$ Rieko Muramatsu, ${ }^{1,2,3}$ Noriko Maedera, ${ }^{1}$ Yoshihisa Koyama, ${ }^{1}$ Machika Hamaguchi, ${ }^{1}$ Harutoshi Fujimura, ${ }^{4}$ \\ Mari Yoshida, ${ }^{5}$ Morichika Konishi, ${ }^{6}$ Nobuyuki Itoh, ${ }^{7}$ Hideki Mochizuki, ${ }^{8}$ and Toshihide Yamashita ${ }^{1,3,9}$ \\ 'Department of Molecular Neuroscience, Graduate School of Medicine, Osaka University, Suita, Osaka, Japan. 2Precursory Research for Embryonic Science and Technology, Japan Science and Technology \\ Agency, Sanbancho, Chiyoda-ku, Tokyo, Japan. ${ }^{3}$ WPI Immunology Frontier Research Center, Osaka University, Suita, Osaka, Japan. ${ }^{4}$ Toneyama National Hospital, Toyonaka, Osaka, Japan. ${ }^{5}$ nnstitute for Medical \\ Science of Aging, Aichi Medical University, Aichi, Japan. ${ }^{6}$ Department of Microbial Chemistry, Kobe Pharmaceutical University, Kobe, Hyogo, Japan. ${ }^{7}$ Department of Genetic Biochemistry, Graduate School of \\ Pharmaceutical Sciences, Kyoto University, Kyoto, Japan. ${ }^{8}$ Department of Neurology, Graduate School of Medicine, and ${ }^{9}$ Graduate School of Frontier Biosciences, Osaka University, Suita, Osaka, Japan.
}

\begin{abstract}
Demyelination in the central nervous system (CNS) leads to severe neurological deficits that can be partially reversed by spontaneous remyelination. Because the CNS is isolated from the peripheral milieu by the blood-brain barrier, remyelination is thought to be controlled by the CNS microenvironment. However, in this work we found that factors derived from peripheral tissue leak into the CNS after injury and promote remyelination in a murine model of toxin-induced demyelination. Mechanistically, leakage of circulating fibroblast growth factor 21 (FGF21), which is predominantly expressed by the pancreas, drives proliferation of oligodendrocyte precursor cells (OPCs) through interactions with $\beta$-klotho, an essential coreceptor of FGF21. We further confirmed that human OPCs expressed $\beta$-klotho and proliferated in response to FGF21 in vitro. Vascular barrier disruption is a common feature of many CNS disorders; thus, our findings reveal a potentially important role for the peripheral milieu in promoting CNS regeneration.
\end{abstract}

\section{Introduction}

Central nervous system (CNS) damage, a hallmark of many CNS disorders, is causatively associated with severe neurological deficits in motor, sensory, cognitive, and other functions (1). Because damaged CNS can spontaneously regenerate after injury, these neurological deficits partially recover over time $(2,3)$. One such regenerative process in the mammalian CNS, remyelination, is initiated by proliferation of oligodendrocyte precursor cells (OPCs), which are distributed widely throughout the mammalian CNS (4-6). OPC proliferation and subsequent remyelination processes (e.g., migration, differentiation into mature oligodendrocytes) ensure the restoration of saltatory conduction (7), provision of trophic support for axons (8), and promotion of functional recovery (9); therefore, the mechanism of remyelination has attracted considerable attention in regard to its potential applications in regenerative medicine aimed at treating CNS demyelinating diseases. Remyelination is thought to be dependent on factors derived from the CNS microenvironment (10, 11). For example, plateletderived growth factor (PDGF) and basic fibroblast growth factor (FGF), which are expressed by astrocytes (10), are both important for OPC proliferation (12). Other studies have shown that factors secreted by vascular cells promote remyelination $(13,14)$. Consistent with these findings, the CNS is isolated from the peripheral milieu by vascular barriers such as the blood-brain barrier (BBB) and blood-spinal cord barrier. To date, the involvement of the peripheral milieu in CNS remyelination has not been elucidated.

Conflict of interest: The authors have declared that no conflict of interest exists. Submitted: March 31, 2017; Accepted: July 6, 2017.

Reference information: J Clin Invest. 2017;127(9):3496-3509.

https://doi.org/10.1172/JCI94337.
Disruption of vascular barriers occurs in several types of disease, including multiple sclerosis, cerebral ischemia, brain tumors, and other neurological diseases (15). Disrupted vascular barriers can lead to hemorrhage, brain hypoperfusion, and transmigration of inflammatory cells into the CNS; consequently, vascular barrier disruption may exacerbate pathological processes (16). However, OPC proliferation increases in proximity to demyelinating lesions (17), which are often characterized by vascular barrier disruption (18). In addition, some of the cells in the CNS express peripheralhormone receptors, such as the insulin and mineralocorticoid receptors, which regulate neurogenesis in the adult CNS $(19,20)$. Although the role of vascular barrier disruption in CNS regeneration has not yet been clarified, we hypothesized that vascular barrier disruption mediated by CNS injury induces the leakage of circulating factors into the CNS, resulting in remyelination.

In this study, we found that circulating FGF21 promotes OPC proliferation. OPC proliferation was elevated in the spinal cords of mice with toxin-induced demyelination, and this proliferation was inhibited by silencing of FGF21 expression in the pancreas. OPCs expressed $\beta$-klotho, an essential coreceptor for FGF21 (21-24), and inhibition of $\beta$-klotho expression in OPCs prevented the increase in OPC proliferation and subsequent remyelination. We also observed FGF21-mediated proliferation of human OPCs, and $\beta$-klotho expression in these cells, in autopsied samples of multiple sclerosis patients.

\section{Results}

OPC proliferation exhibits kinetics similar to those of vascular barrier disruption. We investigated the time changes in vascular permeability and OPC proliferation in the spinal cord following injection of lysophosphatidylcholine (LPC), which perturbs vascular barri- 
er (18) and myelin structures $(14,25)$ with no change of neuronal number (Supplemental Figure 1A; supplemental material available online with this article; https://doi.org/10.1172/JCI94337DS1). Histological analysis revealed the presence of extravasated cadaverine around the site of LPC injection 3 and 7 days after the operation (Figure 1A). In mice that underwent LPC injection, similar kinetics were observed for extravasation of endogenous IgG around the LPC injection site (Figure 1A and Supplemental Figure 1B) and Evans blue leakage into the spinal cord (Figure 1B). Consistent with this, IHC analysis revealed that the number of PDGFR $\alpha^{+} \mathrm{Ki} 67^{+}$cells (proliferating OPCs) around the site of LPC injection increased significantly 3 and 7 days after the operation (Figure 1, C and D). Because OPC proliferation is correlated with remyelination (26, 27), we investigated the change over time in the size of the myelin basic protein-positive $\left(\mathrm{MBP}^{+}\right)$area in the dorsal column of the spinal cord following LPC injection. IHC evaluation revealed loss of myelin in the spinal cord 3 and 7 days after LPC injection; however, this loss was partially restored 7 and 14 days after LPC injection, respectively (Figure 1, E and F). Consistent with this, remyelination after removal of the cuprizone diet, which does not cause BBB disruption $(28,29)$, was less efficient than remyelination after LPC injection (Figure 1, G and H, and Supplemental Figure 1C). These data suggest that vascular barrier disruption is synchronized with OPC proliferation, which precedes remyelination.

Circulating FGF 21 promotes OPC proliferation. Because vascular barrier disruption causes leakage of circulating factors into the CNS (16), we hypothesized that the leaked circulating factors stimulate OPC proliferation, which in turn contributes to remyelination. To test this idea, we collected adult mouse serum and asked whether serum treatment would promote OPC proliferation in vitro. BrdU incorporation assays revealed that serum treatment promoted DNA synthesis in OPCs (Figure 2A and Supplemental Figure 2, A and B), suggesting that adult serum contains an OPC proliferation factor(s). The BrdU incorporation activity was almost completely abolished by heat or Pronase treatment (Figure 2B), indicating that the OPC proliferation factor in adult mouse serum must be a heat-sensitive protein. A pharmacological screen revealed that 2 FGF receptor (FGFR) inhibitors, PD173074 and NF449 $(30,31)$, blocked serum-promoted OPC proliferation (Figure 2, C and D, Supplemental Figure 2C, and Supplemental Table 1), suggesting that the OPC proliferation factor in adult mouse serum belongs to the FGF family. We then sought to determine which FGFRs are involved in serum-promoted OPC proliferation. Knockdown of FGFR1 and FGFR3 in OPCs prevented serum-mediated enhancement of BrdU incorporation (Figure 2E and Supplemental Figure 2D).

As the candidate molecule appears to act on both FGFR1 and FGFR3, we focused on FGF21, which is involved in FGFR1 and FGFR3 signaling $(23,32)$. Because FGFR signaling mediated by FGF21 requires $\beta$-klotho, an essential receptor of FGF21, we asked whether $\beta$-klotho was required for serum-mediated OPC proliferation. $\beta$-Klotho knockdown in OPCs shows lower proliferation activity after serum treatment compared with control OPCs (Figure 2E and Supplemental Figure 2D), and this inhibitory effect was comparable to that in FGFR1 knockdown ( $P$ value for interaction $=$ $0.8828)$ and FGFR3 knockdown ( $P$ value for interaction $=0.855)$. These data indicate that the receptor for FGF21 is involved in serum- mediated OPC proliferation. To test whether FGF21 promotes OPC proliferation directly, we used recombinant FGFs (FGF15 [the mouse ortholog of human FGF19], FGF21, and FGF23), which act as endocrines (33). FGF21 treatment promoted BrdU incorporation into OPCs (Figure 2F), whereas FGF15 and FGF23 did not (Figure 2 F). Moreover, neutralizing antibody against FGF21 abolished the serum-mediated increase in BrdU incorporation by OPCs (Figure $2 \mathrm{G})$. Furthermore, FGF21 did not promote BrdU incorporation in astrocytes (Supplemental Figure 3A). We detected no significant difference in MTT absorbance in OPCs following $\beta$-klotho knockdown (Supplemental Figure 3B). Differentiation into $\mathrm{MBP}^{+}$cells was not altered by $\beta$-klotho knockdown (Supplemental Figure 3C) and FGF21 treatment (Supplemental Figure 3D). We detected no significant difference in OPC proliferation cultured in astrocyte supernatant with or without serum pretreatment (Supplemental Figure 3E). These data suggest that FGF21 in adult mouse serum acts directly as an OPC proliferation factor.

Pancreas-derived FGF21 mediates OPC proliferation. Next, we investigated the expression pattern of FGF21 in adult mice. Quantitative mRNA and protein analysis revealed prominent FGF21 expression in pancreas (Figure 3A), as reported previously (34, 35). IHC analysis revealed abundant FGF21 expression in pancreatic islets, especially in glucagon-producing $\alpha$ cells (Figure 3, B and $\mathrm{C}$ ), which are endocrine cells. To determine whether pancreatic FGF21 is involved in serum-mediated OPC proliferation, we specifically knocked down FGF21 in vivo by transfecting FGF21 siRNA into pancreas (36) (Supplemental Figure 4A), and then collected serum from the transfected mice. BrdU incorporation analysis revealed that serum from pancreas-specific FGF21-knockdown mice exerted weaker proliferative activity than control serum (Figure 3D and Supplemental Figure 4B). These data indicate that pancreas-derived FGF21 is involved in promotion of OPC proliferation mediated by circulating factors in vitro.

Circulating FGF21 drives remyelination. We next asked whether circulating FGF21 promotes OPC proliferation and remyelination in vivo. To test this idea, we monitored the FGF21 level around demyelinating lesions following LPC injection, because leakage of circulating factor into the CNS could be detected around the lesion site with BBB disruption (37). Following LPC injection, FGF21 protein in the spinal cord was present at a higher level than in the control (Figure 4A), although the level of FGF21 mRNA in the spinal cord was unchanged (Supplemental Figure 5A). Fluorescent dye-labeled FGF21 by i.v. administration was detected in the spinal cord of mice after LPC injection (Supplemental Figure 5B). In addition, in FGF21-KO mice, LPC injection caused FGF21 detection in the spinal cord of mice that received recombinant FGF21 intravenously (Supplemental Figure 5C). These results indicate that the elevated FGF21 level around demyelinating lesions is a result of influx from circulating FGF21 into the CNS.

To determine whether in vivo OPC proliferation is promoted by circulating FGF21, we injected LPC into the spinal cords of FGF21-KO mice. The absence of FGF21 prevented the increase in PDGFR $\alpha^{+} \mathrm{Ki}^{+} 7^{+}$cell number around the site of LPC injection (Figure $4 \mathrm{~B})$. Furthermore, the $\mathrm{MBP}^{-}$demyelination area in the dorsal column of the spinal cord was larger in FGF21-KO mice than in control littermates (Figure 4C). Under intact conditions, there was no significant difference between FGF21-KO mice and control lit- 


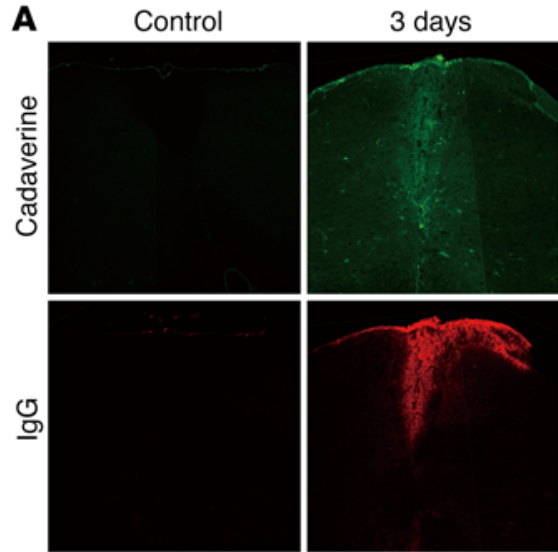

c

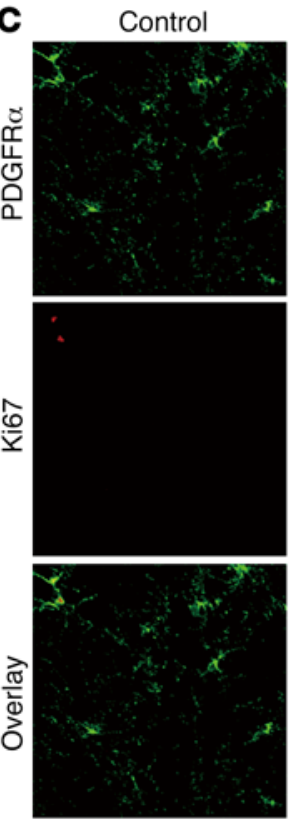

E

Control
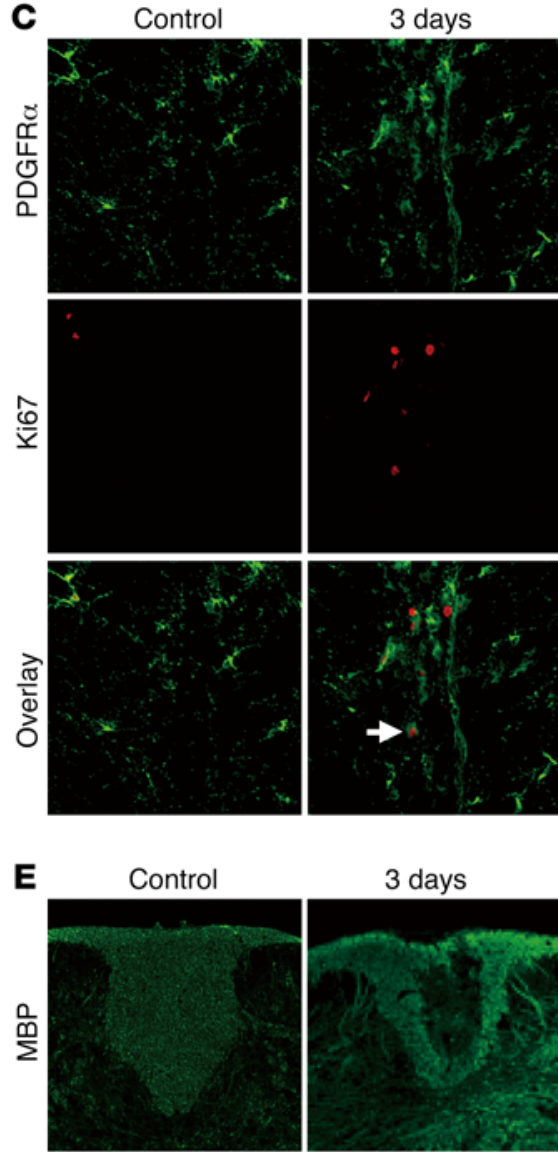

3 days

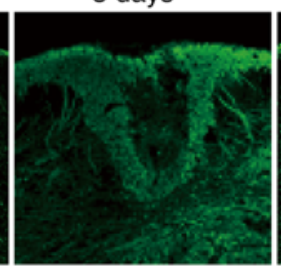

G

Control

3 days

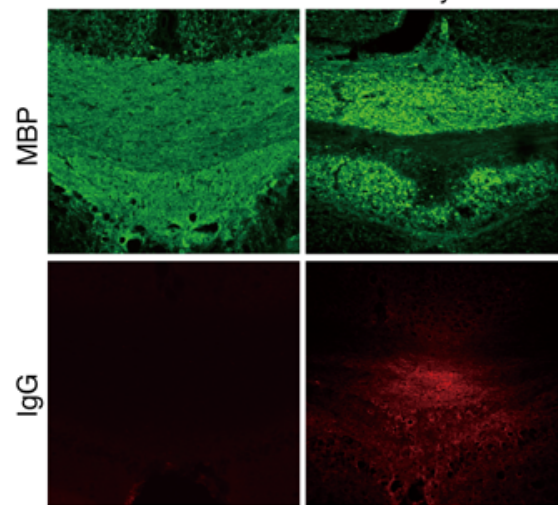

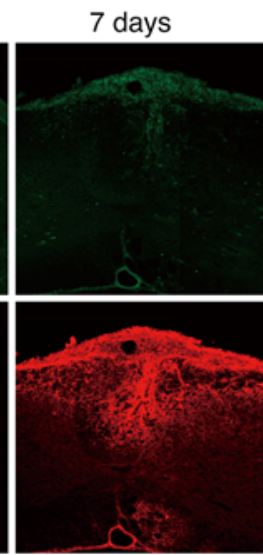

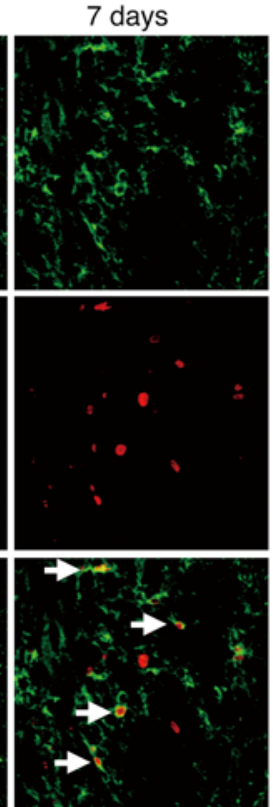

14 days

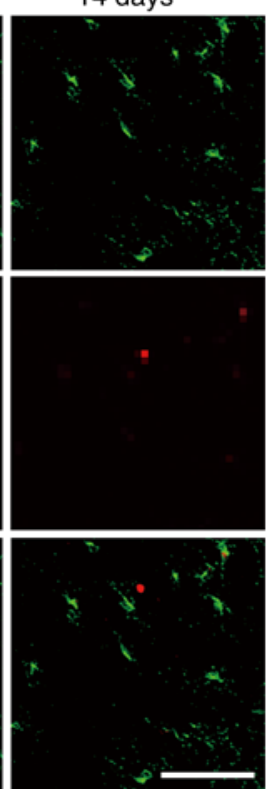

7 days

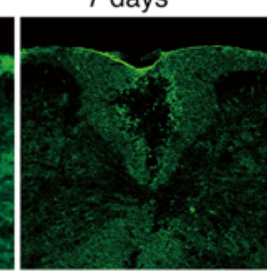

14 days

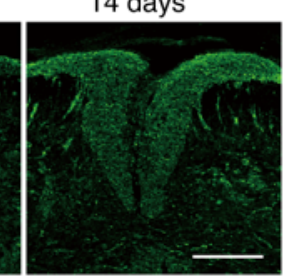

7 days

14 days

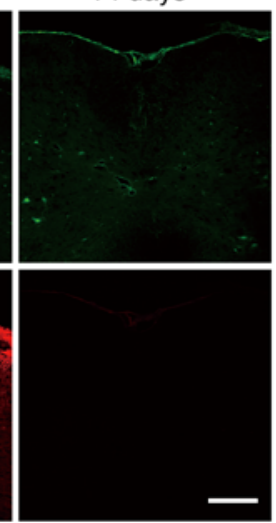

14 days

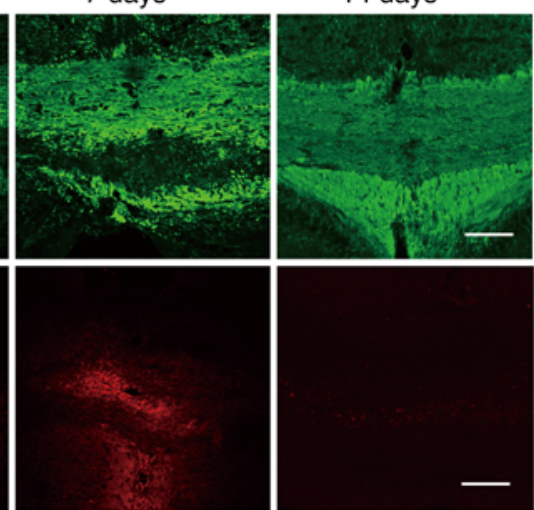

D
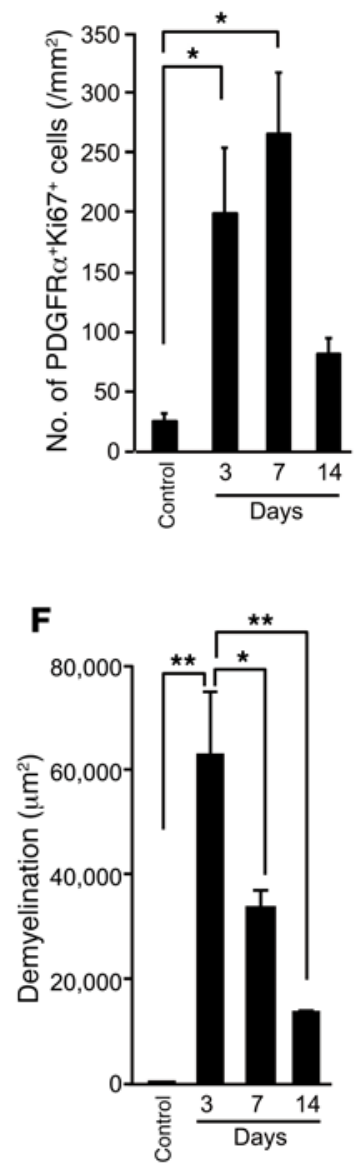

B

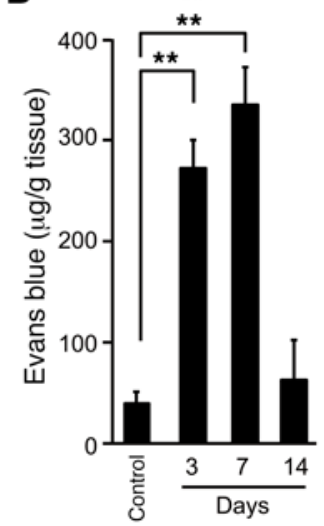

D

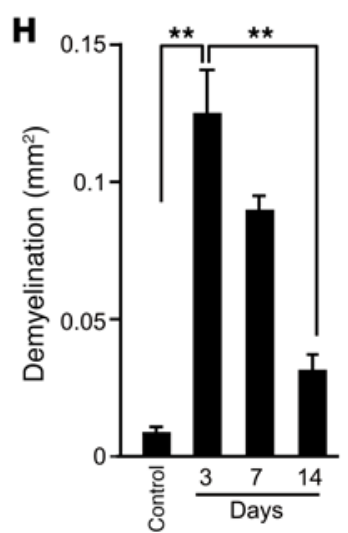


Figure 1. OPC proliferation is promoted under vascular disruption. (A) Representative images of spinal cord sections visualized with Alexa Fluor 488-conjugated cadaverine (top) and endogenous IgG (bottom). Spinal cord sections were prepared at the indicated times after LPC injection. (B) Quantitation of Evans blue leakage in the spinal cord $(n=3)$; ${ }^{* *} P<0.01$. (C) Representative images of spinal cord sections labeled for PDCFR $\alpha$ (top) and Ki67 (middle). Spinal cord sections were prepared at the indicated days after LPC injection. Arrows indicate cells that are colabeled with PDCFR $\alpha$ and Ki67. (D) Graph shows quantitation as indicated in each image ( $n=3$ for control, 3 for d3, 4 for d7, 4 for d14); ${ }^{*} P<0.05$. (E) Representative images of spinal cord sections labeled for MBP. Spinal cord sections were prepared at the indicated days after LPC injection. (F) Graph shows quantitation as indicated in each image ( $n=3$ for control, 3 for d3, 3 for d7, 4 for d14); $P=0.002,0.0424,0.0018$ (left to right). ${ }^{*} P<0.05,{ }^{* *} P<0.01$. (C) Representative images of brain sections visualized with MBP (top) and endogenous IgC (bottom) the indicated number of days after LPC injection. (H) Graph shows the time course of demyelination after LPC injection ( $n=3$ for control, 6 for d3, 7 for d7, 4 for d14); ${ }^{*} P<0.05,{ }^{*} P<0.01$, determined by ANOVA with Tukey's post hoc test or Dunnett's test. Error bars represent SEM. Scale bars: $200 \mu \mathrm{m}$ (A and E); $50 \mu \mathrm{m}$ (C); $100 \mu \mathrm{m}$ (C).

termates in PDGFR $\alpha^{+} \mathrm{OPC}$ number, PDGFR $\alpha^{+} \mathrm{BrdU}^{+}$cell number, or myelin formation (Supplemental Figure 5, D-F). We confirmed that FGF21-KO mice did not exhibit a significant increase in demyelination after LPC injection (Supplemental Figure 6A). There was no significant difference between groups in $\mathrm{GFAP}^{+}$cell number in the spinal cord 7 days after LPC injection (Supplemental Figure $6 \mathrm{~B})$. These data imply that peripheral tissue-derived FGF21 promotes OPC proliferation in areas lacking a vascular barrier.

To confirm that these observations rely on the degree of structural remyelination, we calculated the $g$-ratio, which is positively correlated with the thickness of myelin sheaths. A typical $g$-ratio for a normally myelinated axon is between 0.6 and 0.8 , whereas a $g$-ratio of 1.0 indicates complete demyelination. FGF21-knockdown mice exhibited few signs of remyelination relative to controls (Figure 4D). These data indicate that circulating FGF21 also promotes structural remyelination.

Spinal cord demyelination causes severe motor deficits, which recover in a time-dependent manner as a result of spontaneous remyelination $(14,38)$. Therefore, we speculated that peripheral FGF21 is involved in improvement of motor function. Behavioral evaluation by ladder-walk test revealed that recovery from hind limb motor dysfunction due to LPC injection was inhibited in FGF21-KO mice (Figure 4E). These data indicate that the activity of circulating FGF21 encompasses neurological function.

We also investigated the possibility that circulating FGF21 acts directly on remyelination in vivo. To this end, we injected LPC into the spinal cord of FGF21-KO mice, with or without intrathecal FGF21 treatment. IHC analysis revealed that the mice subjected to intrathecal administration of FGF21 had reduced demyelination around the site of LPC injection, relative to control mice (i.e., mice not subjected to FGF21 treatment) (Figure 4F). We also found that FGF21 treatment promoted reduction of demyelination around the site of LPC injection in WT mice (Figure $4 \mathrm{G}$ ). These data raise the possibility that circulating FGF21 acts directly on OPCs in the $\mathrm{CNS}$, resulting in remyelination.

$\beta$-Klotho in OPCs is involved in remyelination. We next investigated the possibility that circulating FGF21 acts directly on
OPC proliferation in vivo. Because FGF21-mediated signaling is required for the expression of $\beta$-klotho (21-24), which forms a complex with FGFRs, we examined $\beta$-klotho expression in OPCs after LPC injection. IHC analysis revealed that $\beta$-klotho expression in PDGFR $\alpha^{+}$OPCs was elevated after LPC injection (Figure $5 \mathrm{~A}$ and Supplemental Figure 7A). $\beta$-Klotho expression in proliferating OPCs was higher than that in quiescent OPCs (Supplemental Figure 7B). $\beta$-Klotho expression was also detected in $\mathrm{O}^{+}$immature and mature oligodendrocytes and in Adenomatous polyposis coli (APC)-positive mature oligodendrocytes in the spinal cord after LPC injection (Supplemental Figure 7C).

To determine whether OPC proliferation depends on $\beta$-klotho in OPCs, we generated a conditional knockout mouse in which $\beta$-klotho is knocked down specifically in PDGFR $\alpha^{+}$OPCs $\left(\mathrm{Kl}^{\text {fl/fl}}\right.$ Pdgfra-CreERT mice; Supplemental Figure 7D). We conducted LPC injection into the spinal cord of the conditional knockout mice and performed histological analysis to evaluate OPC number and demyelination area in the spinal cord. There were fewer PDGFR $\alpha^{+}$ cells in the spinal cords of conditional knockout mice than in spinal cords of control mice 7 days after LPC injection (Figure 5B). These conditional knockout mice also had a larger $\mathrm{MBP}^{-}$area in the dorsal spinal cord than control littermates (Figure 5C), indicating that $\beta$-klotho expression in OPCs is involved in OPC proliferation and subsequent remyelination. We also found that FGF21 administration did not promote remyelination in $\beta$-klotho-floxed mice (Supplemental Figure 8A), further supporting the critical role of $\beta$-klotho in the signal transduction of FGF21. These data provide the functional link of FGF21 and $\beta$-klotho in remyelination.

We next asked whether FGF21-mediated remyelination was restricted to LPC-mediated demyelination. Because demyelination is a common feature of many CNS disorders, including traumatic brain injury (39), we conducted controlled cortical injury to evaluate the suitability of FGF21 as a therapeutic target with broad pathological significance. Intracerebroventricular administration of FGF21 increased the number of PDGFR $\alpha^{+} \mathrm{Ki} 67^{+}$cells around the lesion 7 days after brain injury (Figure 5D and Supplemental Figure $8 \mathrm{~B}$ ). The $\mathrm{MBP}^{-}$area in the corpus callosum was smaller in FGF21-treated mice than in control mice (Figure 5E). Furthermore, electron microscopy also revealed an increase in structural remyelination by FGF21 treatment after brain injury (Figure 5F). These results suggest that FGF21 promotes remyelination in many CNS pathological states that are characterized by demyelination.

Finally, we confirmed that our findings may apply to humans. IHC analysis revealed enhanced $\beta$-klotho expression in PDGFR $\alpha^{+}$ OPCs in the spinal cord tissues from individuals with multiple sclerosis (Figure 6A). We also found that treatment with recombinant human FGF21 increased BrdU incorporation in human OPCs in vitro (Figure 6B), implying that FGF21-mediated OPC proliferation is conserved in humans.

\section{Discussion}

Peripheral tissue-derived factors promote OPC proliferation in the CNS (Supplemental Figure 9). Our findings suggest a novel paradigm for the mechanism of CNS regeneration, which has previously been considered to primarily involve the CNS microenvironment. Because OPC proliferation is just one process of CNS regeneration, the influence of peripheral tissue-derived factors on other regen- 
A

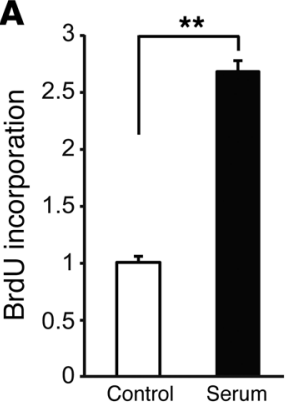

B

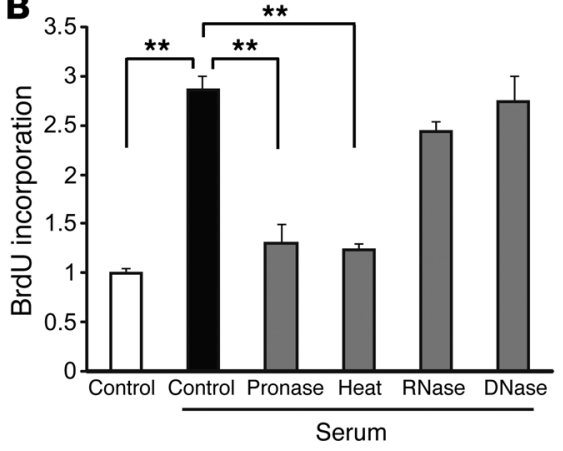

C

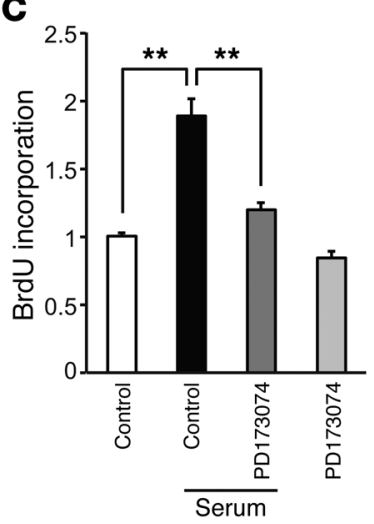

D

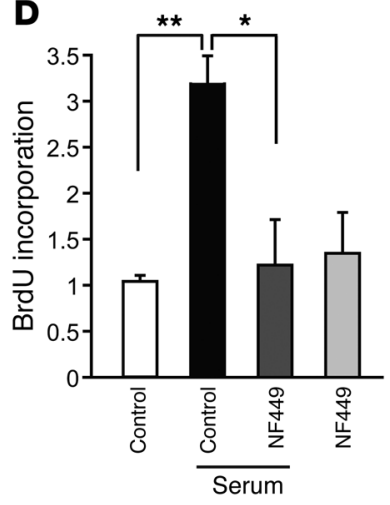

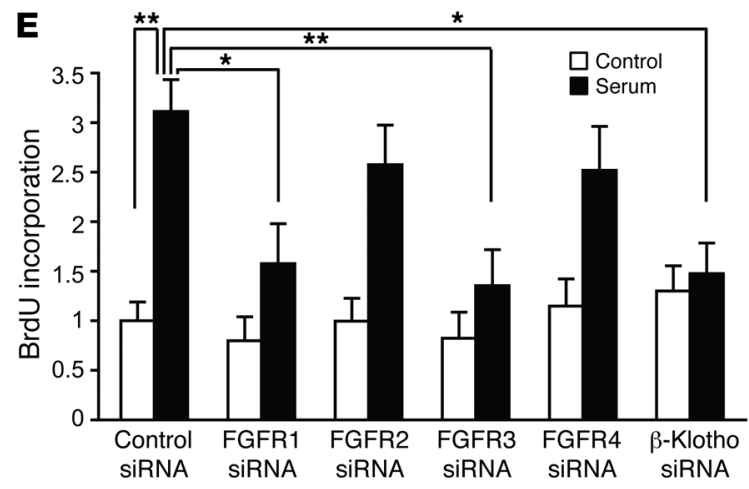

$\mathbf{F}$

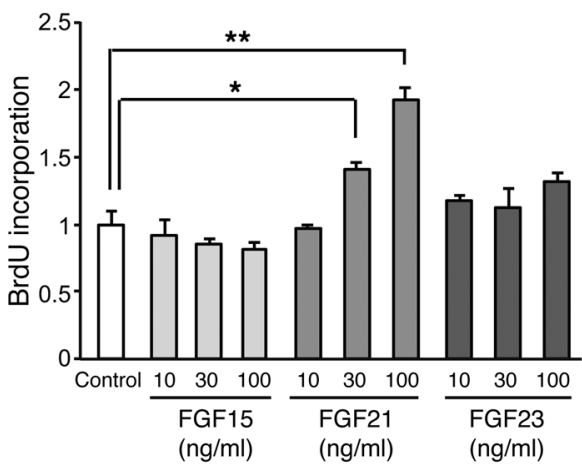

G

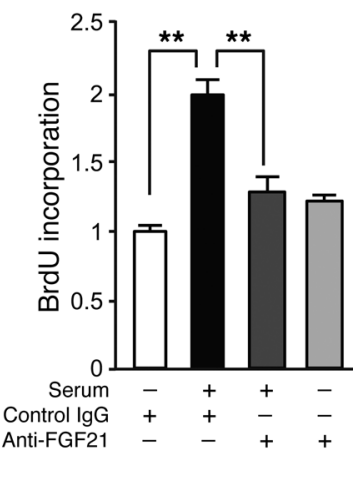

Figure 2. Circulating FGF21 promotes OPC proliferation. (A) Concentration-dependence of BrdU incorporation in $\mathrm{A}_{2} \mathrm{~B} 5^{+} \mathrm{OPCs}$ cultured with adult mice serum $(n=6)$. (B) BrdU incorporation in A2B5+ OPCs 1 day after stimulation with adult mouse serum heated or pretreated with the indicated reagents $(n=6)$. (C) BrdU incorporation in OPCs after serum stimulation with PD173074 $(10 \mathrm{nM})$, an inhibitor of FGFR $(n=4)$. (D) BrdU incorporation in OPCs after serum stimulation with NF449 $(10 \mu \mathrm{M})$, an inhibitor of FGFR3 $(n=4)$. (E) BrdU incorporation in mouse OPCs with FGFR and $\beta$-klotho knockdown after serum stimulation $(n=7)$. (F) BrdU incorporation in OPCs after stimulation with recombinant FGF15, FGF21, and FGF23 $(n=4)$. (G) BrdU incorporation in OPCs after serum stimulation with neutralizing antibody against FGF21 $(n=5)$, determined by Student's $t$ test or by ANOVA with Tukey's post hoc test or Dunnett's test. Error bars represent SEM. ${ }^{*} P<0.05,{ }^{*} P<0.01$.

eration mechanisms, including oligodendrocyte development and neuronal plasticity, remains an open question. Regarding the duration of vascular barrier disruption after CNS injury, we observed that vascular permeability was significantly recovered 14 days after LPC injection, relative to 7 days after injection. These kinetics observations are supported by other reports that restoration of vascular barrier in CNS lesions begins within a week after injury (40, 41). By contrast, our in vivo experiment revealed extension of the MBP-expressing area 2 weeks after LPC injection. Considering the relative kinetics of vascular barrier disruption and remyelination, it is conceivable that peripheral tissue-derived factors play a strongly supportive role during early regeneration mechanisms such as OPC proliferation, at a time when there is abundant extravasation of blood components into the CNS.

In this context, we found that FGF21, which is predominantly expressed from pancreas, is crucial for peripheral tissuemediated OPC proliferation. The results of this study reveal an unexpected role of FGF21, which has been previously characterized as a metabolic regulator (42). In reviewing previous findings regarding FGF21 function in the CNS, we noted that FGF21 can cross the BBB (43), but the FGF21 level in the cerebrospinal fluid of healthy patients is approximately $40 \%$ of that in the plasma (44).
Thus, CNS entry of peripheral FGF21 is limited in normal adult subjects. Indeed, we observed no significant difference in OPC number between WT and Fgf21-KO mice, indicating that FGF21 does not control OPC proliferation under normal conditions. In the CNS, FGF21 regulates metabolism, circadian behavior, and neuroendocrine control of female reproduction via direct action on hypothalamus $(32,45)$, which structurally lacks a BBB (46). This anatomical feature also supports our hypothesis: peripheral FGF21 acts on the CNS directly under certain situations, such as pathological lesions with vascular barrier disruption or normal tissues with high vascular permeability.

Our culture experiments suggested that $\beta$-klotho is involved in OPC proliferation. $\beta$-Klotho is widely believed to be a simple coreceptor required for FGF21 binding to FGFRs (21-24), which are involved in signal transduction. All FGFRs mediate cell proliferation (47), and conditional knockout mice lacking FGFR1/FGFR2 in OPCs show decreased oligodendrocyte repopulation in a chronic demyelination model (48). This finding is consistent with our results that FGFR1 is involved in serum-mediated OPC proliferation. Regarding FGFR3, FGFR3-null mice exhibit reduced oligodendrocyte number during development, whereas this change is not due to reduction of oligodendrocyte progenitor proliferation 

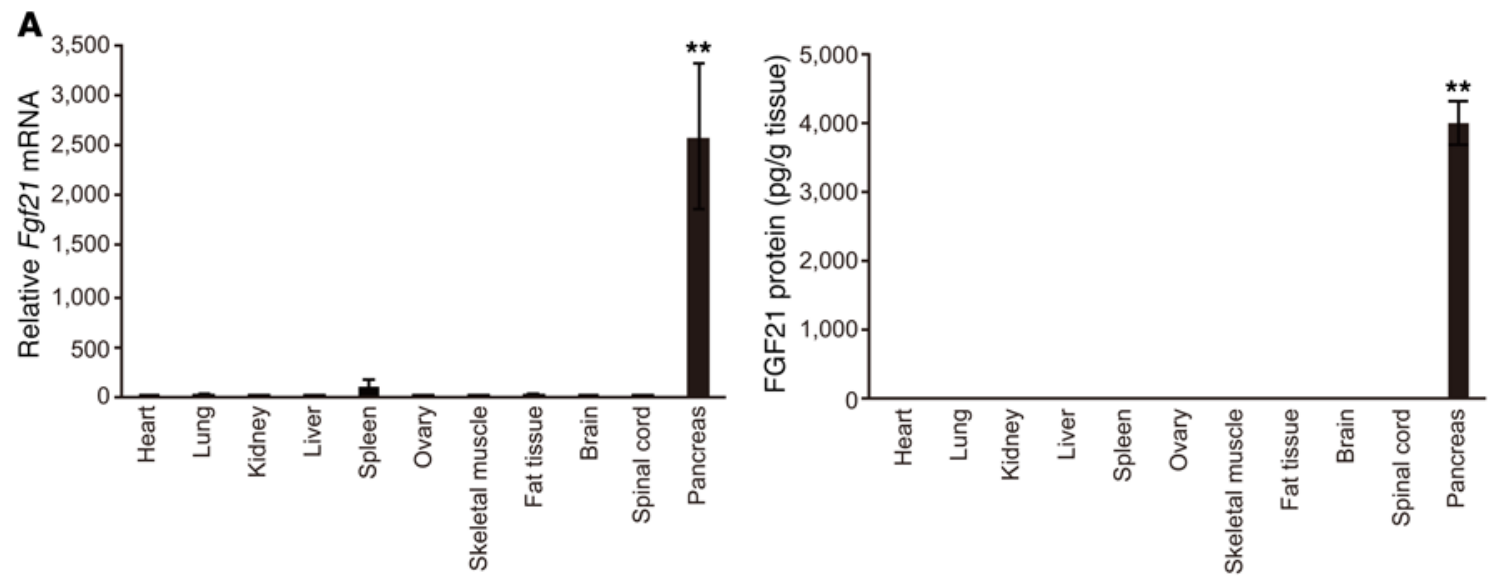
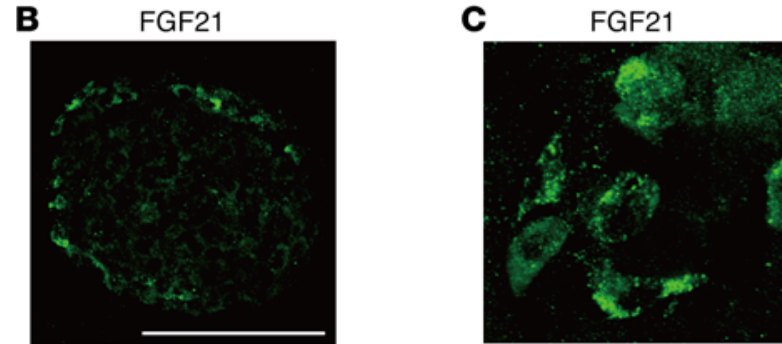

FGF21

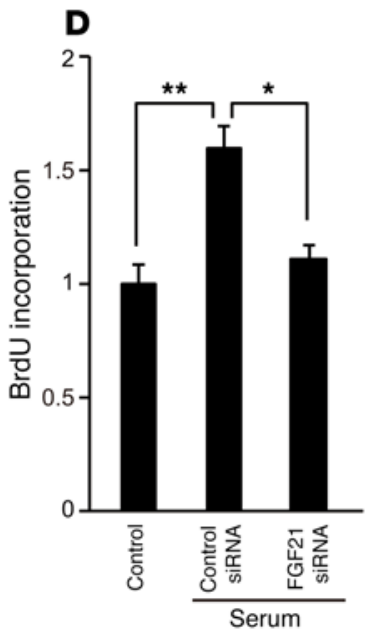

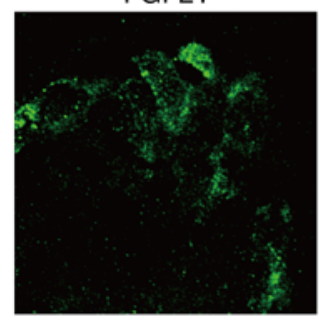

FGF21

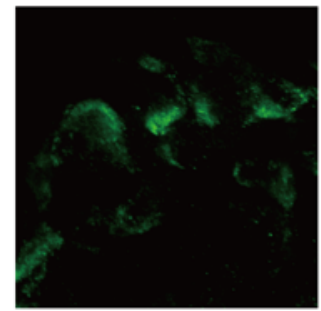

Glucagon

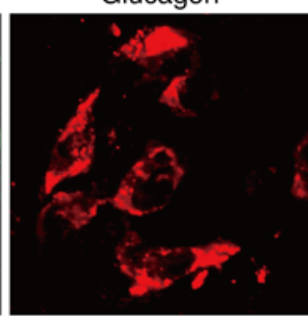

Insulin

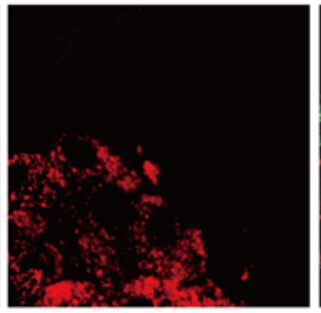

Somatostatin

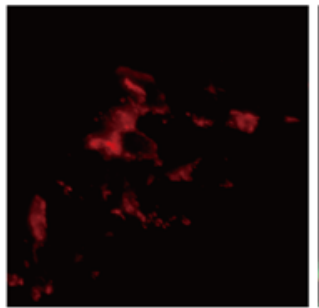

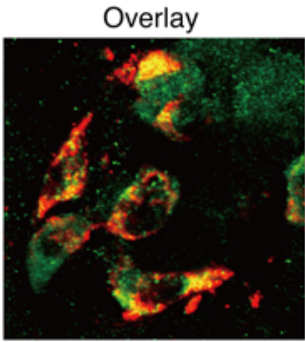

Overlay

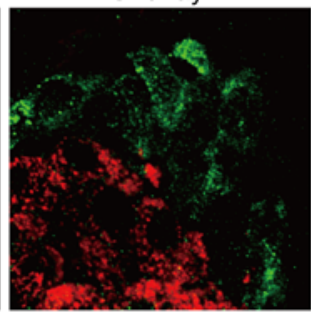

Overlay

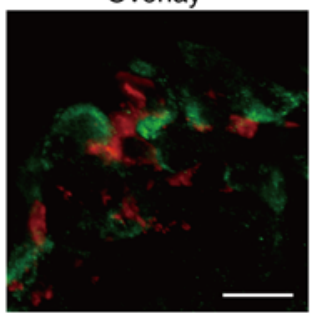

Figure 3. FGF21 secreted from pancreas induces OPC proliferation. (A) Quantitations of Fgf21 mRNA (left) and FGF21 protein (right) in individual organs of intact mice $(n=9)$; ${ }^{* *} P<0.01$. (B) Representative images of FGF21-immunolabeled pancreas of intact mice $(n=3)$. (C) Double IHC staining for FGF21 with the indicated cell markers in the pancreas of adult mice $(n=3)$. (D) BrdU incorporation in mouse OPCs after stimulation with serum from mice with FGF21 knockdown in the pancreas $(n=4) ;{ }^{*} P<0.05$, ${ }^{* *} P<0.01$, as determined by ANOVA with Tukey's post hoc test. Error bars represent SEM. Scale bars: $50 \mu \mathrm{m}(\mathbf{B}) ; 10 \mu \mathrm{m}$ (C).

(49). As developmental myelination occurs at postnatal weeks 2-3 when the BBB is already formed, FGF21 in blood does not penetrate into the CNS, and therefore FGF21 level in the CNS should be much lower than that in the blood. In contrast, our in vitro experiments mimic the pathological condition in the CNS that suffered exposure of blood components (including FGF21) by disruption of $\mathrm{BBB}$, which is formed during the embryonic stage. The seemingly contradictory findings in the effects of FGFR3 inhibition on OPC proliferation during the developmental stage in vivo and in our in vitro condition may be due to the absence or presence of ligands for FGFR3, including FGF21. We note that FGFR1 and FGFR3 are expressed in OPCs in vivo (50-52), and our in vitro experiments reveal that serum-mediated OPC proliferation is dependent on FGFR1 and FGFR3. In addition, FGFR3 expression in the white matter of the spinal cord is elevated in the experimental demyelinating disease (53), and FGFR3 is considered to promote proliferation of oligodendrocyte progenitors (54). Therefore, we do not exclude the possibility that FGFR3 might be another component necessary for the signal transduction of FGF21 specifically in the pathological condition in the CNS. 
A
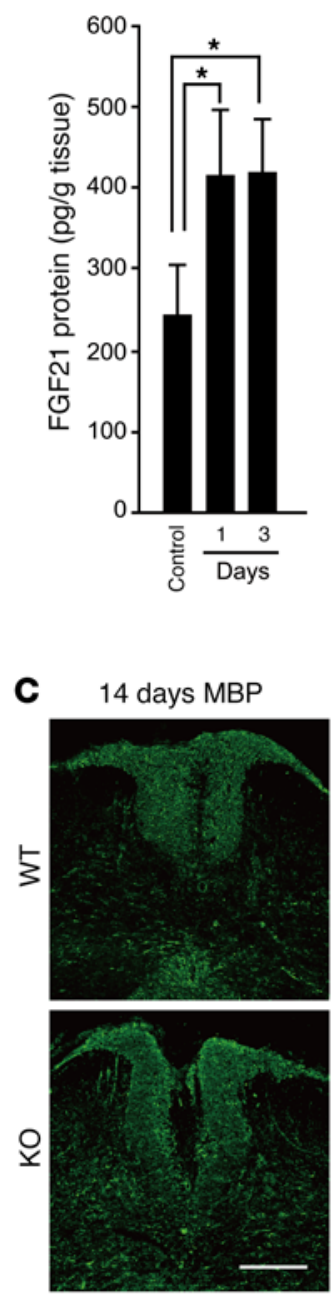

$\mathbf{F}$

14 days MBP
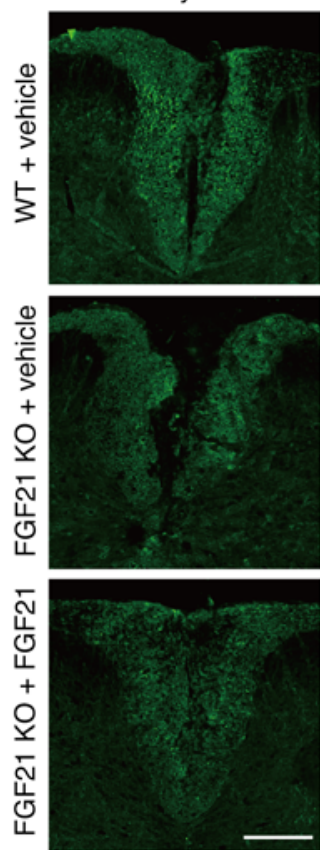

B
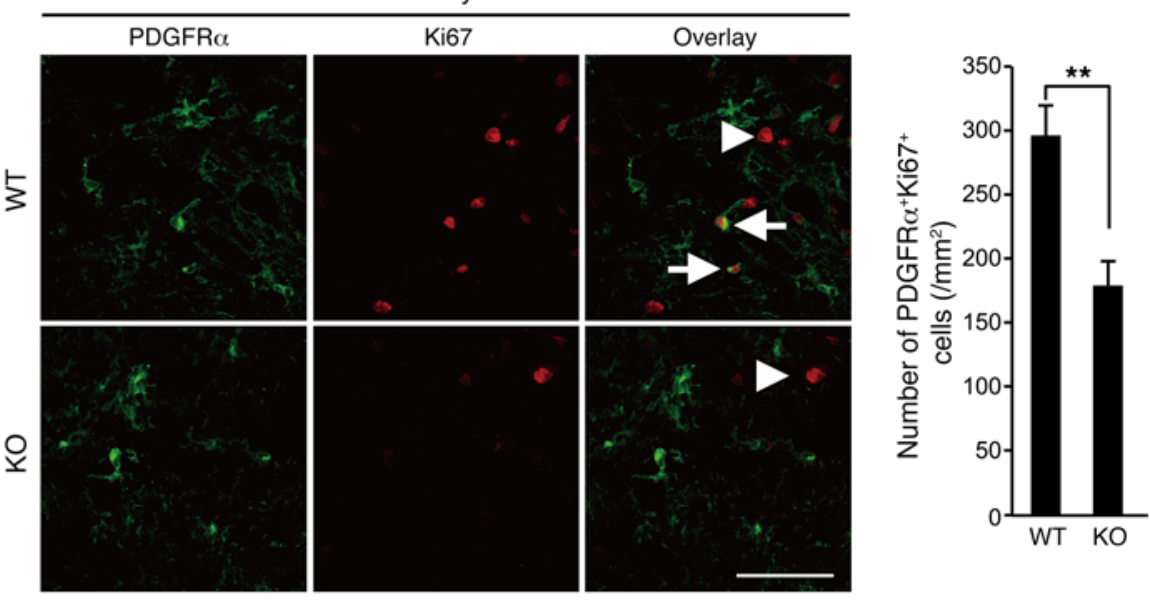

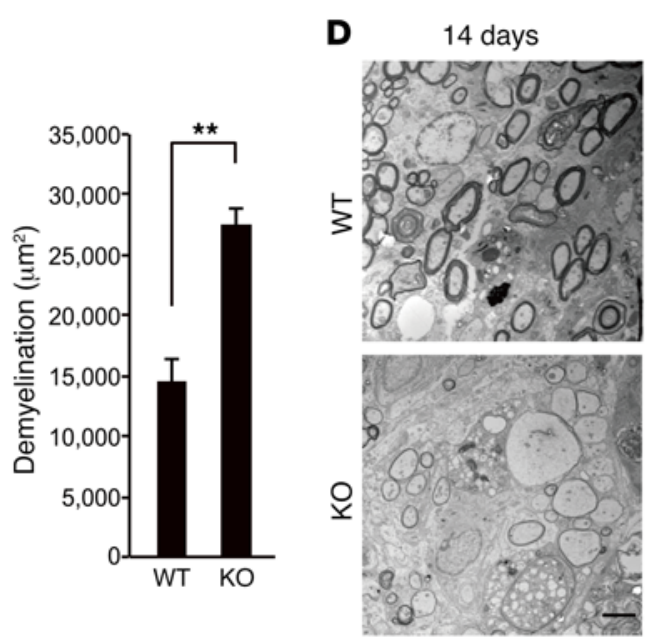

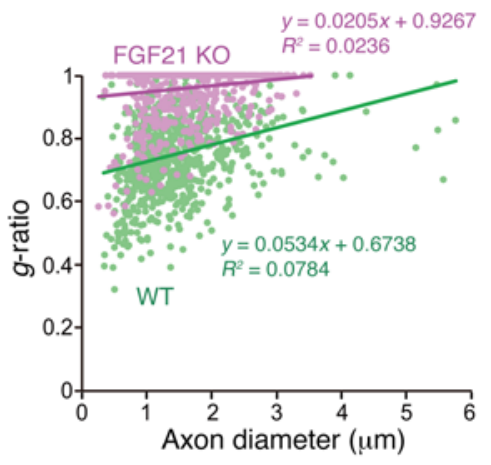
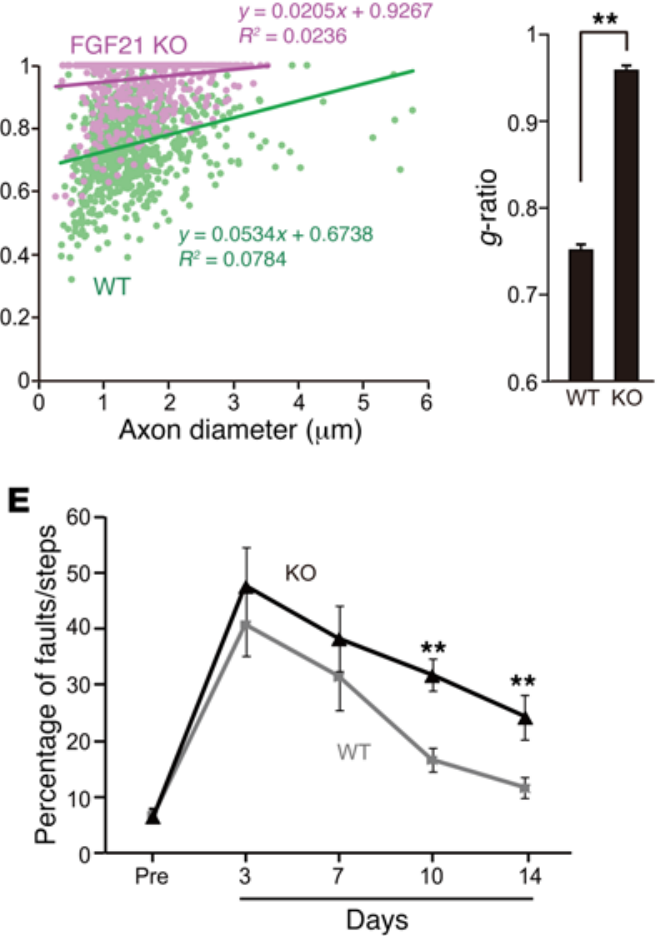
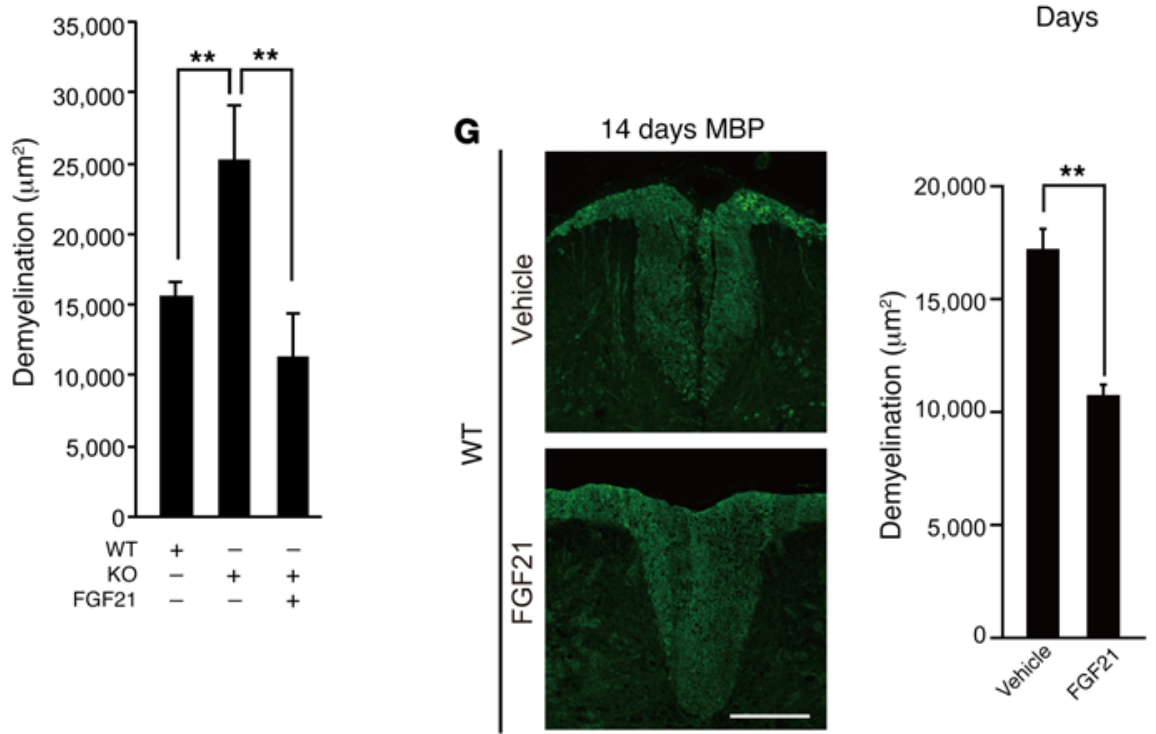
Figure 4. Circulating FGF21 drives OPC proliferation. (A) Quantitation of FGF21 protein in the spinal cord 1 day and 3 days after LPC injection ( $n=5$ for control, 5 for d1, 4 for d3). (B) Representative images of spinal cord sections double-labeled for PDGFR $\alpha$ and Ki67. Sections were obtained from FGF21-KO mice and control littermates 7 days after LPC injection. Graph shows quantitations as indicated in the images $(n=5)$. Arrows indicate $\mathrm{Ki}^{\circ} 7^{+}$cells colabeled with PDCFR $\alpha$; arrowheads indicate $\mathrm{Ki}^{+} 7^{+}$ cells not labeled with PDCFR $\alpha$. (C) Representative images of spinal cord sections labeled for MBP. Sections were obtained from mouse spinal cord 14 days after LPC injection. Graph shows quantitations as indicated in the images $(n=5)$. (D) Representative immunoelectron microscopy images of myelin in the spinal cord. Sections were obtained from FGF21-KO mice and control littermates 14 days after LPC injection. Graphs show quantitations of $g$-ratio indicated in the images $(n=3)$. (E) Motor function was assessed by ladder-walk test ( $n=11$ for control littermates, 9 for FGF21-KO mice). (F) Representative images of spinal cord sections labeled for MBP. Graph shows quantitations as indicated in the images $(n=5$ for control littermates + vehicle, 5 for FGF21-KO mice + vehicle, 4 for FGF21-KO mice + FGF21). (G) Representative images of spinal cord sections labeled for MBP. Graph shows quantitations as indicated in the images $(n=4) ;{ }^{* *} P<0.01$ as determined by Student's $t$ test or by ANOVA with Tukey's post hoc test or Dunnett's test. ${ }^{*} P<0.05,{ }^{*} P<0.01$. Error bars represent SEM. Scale bars: $50 \mu \mathrm{m}$ (B); $200 \mu \mathrm{m}$ (C, F, and C); $2 \mu \mathrm{m}$ (D).

We should note that FGF21-mediated OPC proliferation is one of the mechanisms of remyelination. In terms of molecular mechanism, we just focused on the direct action of FGF21 on OPC proliferation; however, FGF also regulates expression of VEGF receptor 2 (55). Because VEGF signaling is related to brain homeostasis, including OPC migration, a process that involves remyelination $(56,57)$, an indirect effect of FGF21 on OPCs may also contribute to oligodendrocyte development and remyelination. There is also some possibility that other molecules, which are not targeted by the inhibitors that use pharmacological screening, are involved in the serum-mediated OPC proliferation. Future studies should seek to reveal novel molecular mechanisms of remyelination based on the concepts proposed in this study.

Meanwhile, FGF21-associated drugs for treating diabetes have recently been developed by pharmaceutical companies, and some of these compounds have reached the stage of clinical trials (58). In this study, we observed that FGF21 treatment also promotes human OPC proliferation. Therefore, we believe that these FGF21-associated drugs may exert FGF21-mediated remyelination effect and provide clinical benefits in patients with CNS demyelination.

The findings of this study emphasize the novel concept that the peripheral milieu controls CNS regeneration. It is widely believed that CNS regeneration occurs rarely in adults because of the age-dependent decline in regenerative capacity (2), which can be partially explained by a reduction in cell-intrinsic plasticity (59). Several recent studies suggested that age-related decline of neuronal plasticity depends on an aged systemic milieu (60-62). The results of these studies predict that a young peripheral milieu should enhance regenerative capacity in the adult CNS. Although our study is currently limited to focusing on a circulating molecule in the adult systemic milieu, infiltrating peripheral macrophages in the youthful systemic environment are known to enhance spinal cord remyelination (17). Future studies focusing on the young systemic milieu may contribute to the development of methods for CNS regenerative medicine.

\section{Methods}

Mice. All experimental procedures were approved by the Institutional Animal Care Committee of Osaka University. All experiments were run in a blinded fashion. Female C57BL/6J mice were obtained from Charles River Japan, Japan SLC, or CLEA Japan. Fgf21-KO mice (C57BL/6 background) were previously described (63). Pdgfra-CreERT mice (C57BL/6 background) were purchased from the Jackson Laboratory. $\beta$-Klotho-floxed mice (Kl$b^{f / f l}$; C57BL/6 background) were provided by Steven Kliewer (University of Texas Southwestern Medical Center). Mice were housed in an air-conditioned room at $23^{\circ} \mathrm{C} \pm 1^{\circ} \mathrm{C}$ with a 12-hour light-dark cycle, and had free access to water and food.

The OPC-specific $\beta$-klotho deletion mice were obtained by crossing of the $\beta$-klotho-floxed mice with the Pdgfra-CreERT mice. Cre recombination in the generated mice (7- 8 weeks) was induced by administration of tamoxifen ( $75 \mathrm{mg} / \mathrm{kg}$, i.p.; Sigma-Aldrich) daily for 5 consecutive days. Ten days after the first tamoxifen administration, mRNA in PDGFR $\alpha^{+}$cells in brain was obtained from the Cre/-::flox/ flox mice and -/-::flox/flox mice to assess the efficiency of $\beta$-klotho deletion. Relative $\beta$-klotho expression in PDGFR $\alpha$-labeled cells was assessed by immunohistological analysis.

Surgical procedure. Focal demyelination in the brain was induced by stereotactic infusion of $1 \%$ (wt/vol) lysophosphatidylcholine (LPC, Sigma) dissolved in PBS. LPC was slowly injected into the corpus callosum of adult female mice ( $2 \mathrm{~mm}$ posterior to bregma, midline, depth of $2 \mathrm{~mm}, 2 \mu \mathrm{l}$ per site) using a glass capillary attached to a microsyringe.

To induce demyelination in the spinal cord, adult female mice underwent laminectomy at Th11-12 and received injections of $2 \mu \mathrm{l}$ of $1 \%$ (wt/vol) LPC in the dorsal column midline at a depth of $0.5 \mathrm{~mm}$. For administration of recombinant FGF21, a cannula from an Alzet osmotic pump (model 1007D, Alzet Corp.) was placed under the dura at the thoracic spinal cord immediately after LPC injection. The pump was filled with vehicle solution (PBS containing 0.5\% BSA) or recombinant mouse FGF21 (50 ng/kg of body weight per day for 1 week) and implanted s.c. into the back. Administration was continued until the end of the study.

To create traumatic brain injury, adult female mice were stabilized in a stereotaxic frame (Narishige) after deep anesthesia. A midline incision was made in the scalp, and the fascia was retracted to expose the cranium. A circular craniotomy opening $4 \mathrm{~mm}$ in diameter was created on the left side, with the center at $0 \mathrm{~mm}$ anteroposterior and $2 \mathrm{~mm}$ lateral to the bregma. Controlled cortical impact was produced with a Pneumatic Impact Device (AmScien Instruments) using a 3-mm-diameter flat-tip, as described previously (64). The impact parameters were $4.0-4.5 \mathrm{~mm} / \mathrm{ms}$ velocity, $1 \mathrm{~mm}$ depth, and $120 \mathrm{~ms}$ duration time. The scalp was then sutured and closed, and the mice were left to wake from the anesthesia. For infusion of recombinant FGF21, a 28-gauge stainless steel cannula connected to an Alzet osmotic pump (model 1002, Alzet Corp.) was guided to the lateral ventricle (coordinates: $0 \mathrm{~mm}$ bregma, $1 \mathrm{~mm}$ lateral, and $2.5 \mathrm{~mm}$ ventral). The pump was filled with vehicle solution (PBS containing $0.5 \%$ BSA) or recombinant mouse FGF21 (50 ng/ $\mathrm{kg}$ of body weight per day for 2 weeks) and implanted s.c. into the back.

Demyelination by cuprizone diet. Mice were placed on a diet of $0.2 \%$ (wt/wt) cuprizone mixed into chow pellets. Mice were exposed to continuous cuprizone feeding for 12 weeks, and then returned to normal chow pellets. Mice were maintained in sterile, pathogen-free conditions. 


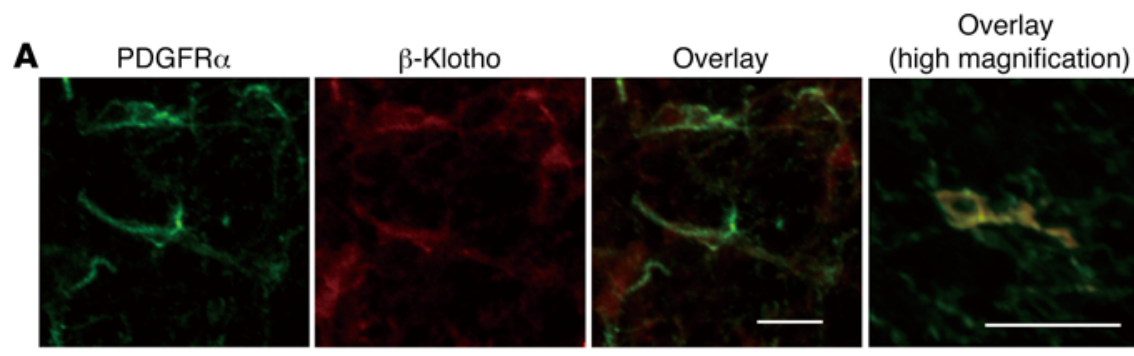

B
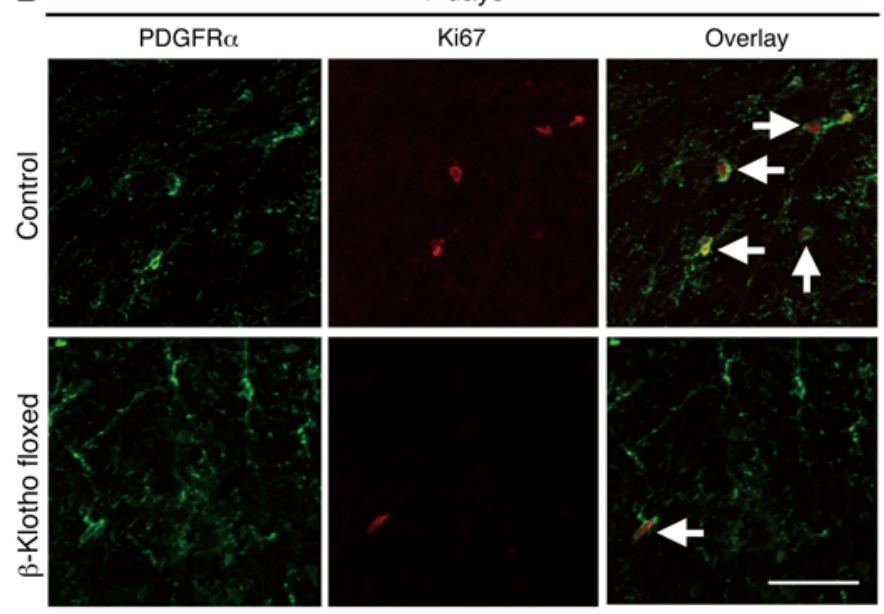

D
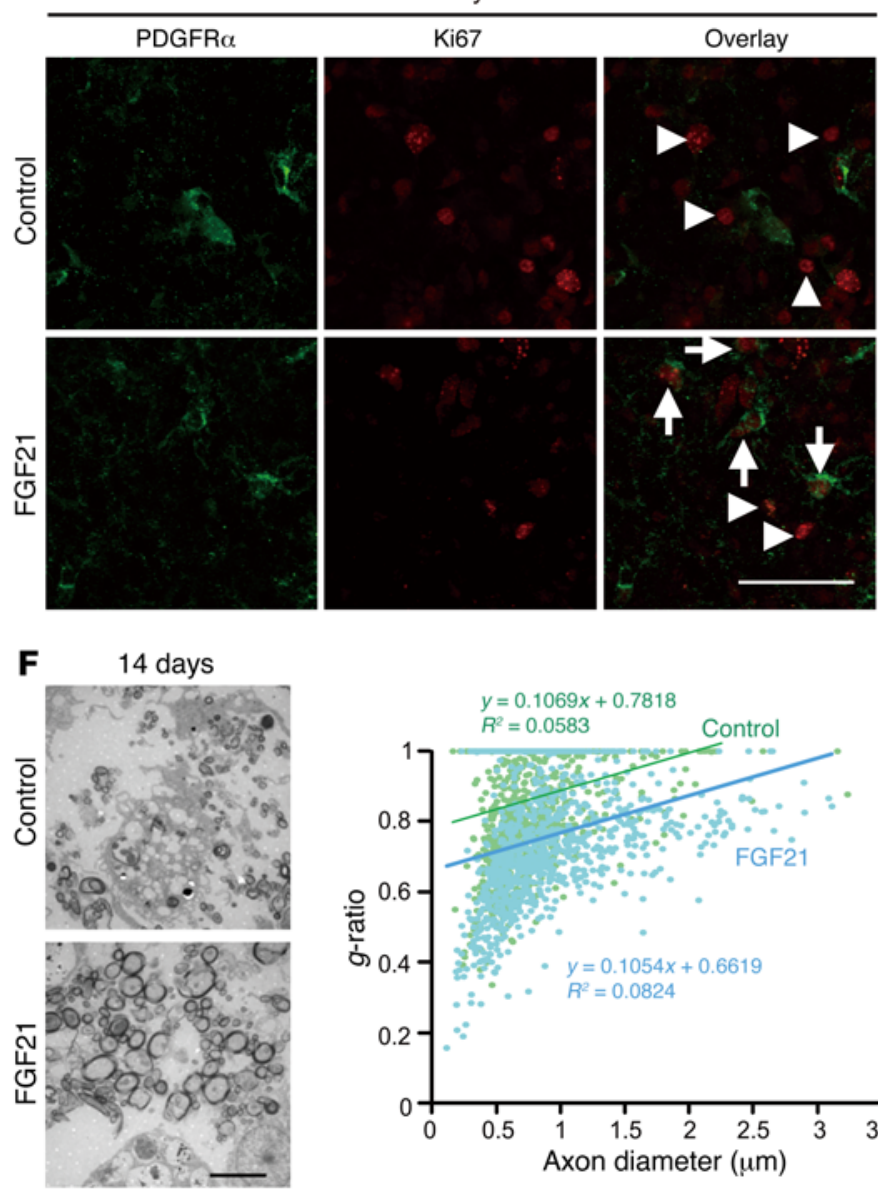

$\frac{7 \text { days }}{\text { Ki67 }}$

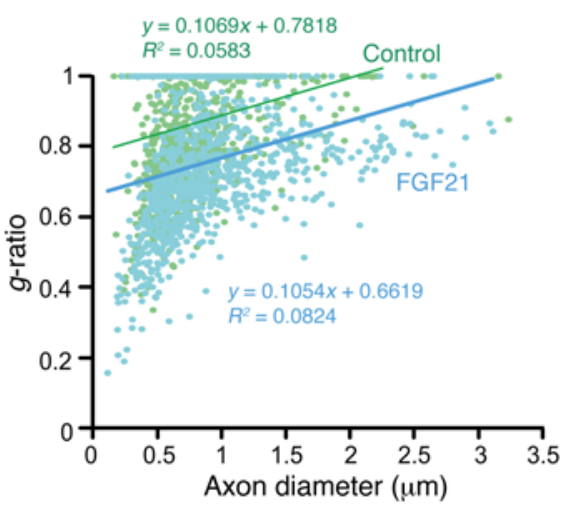

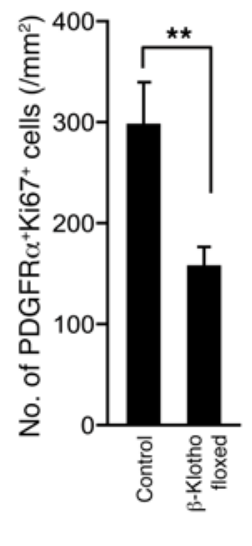

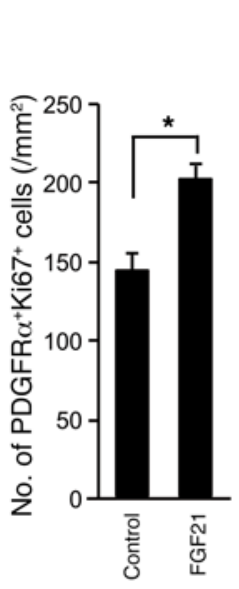

E
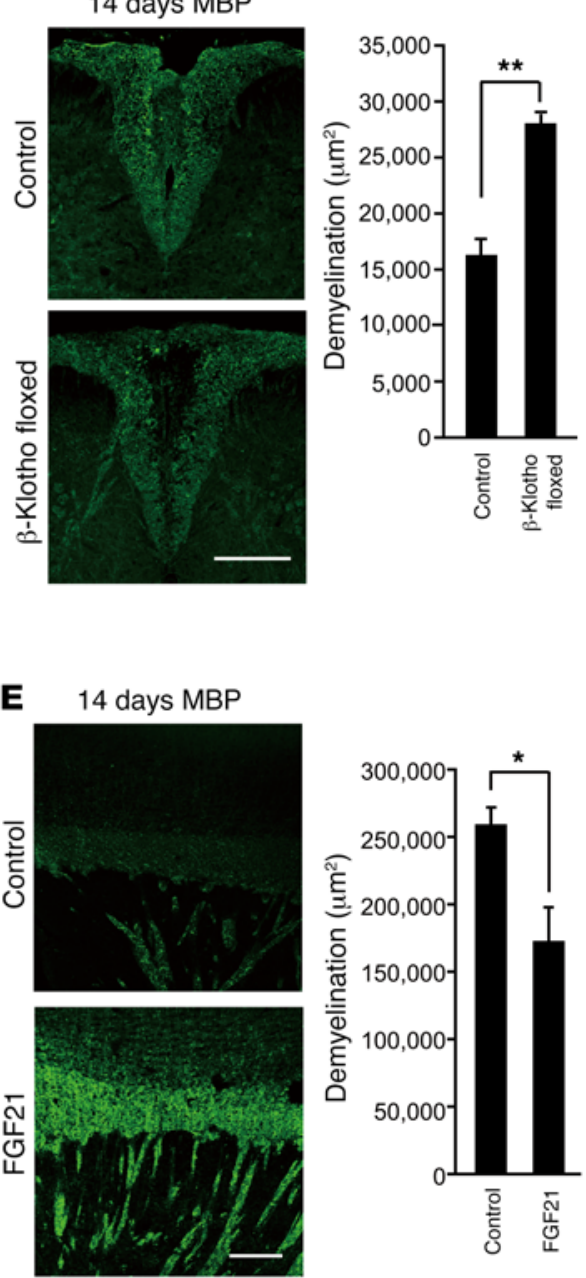

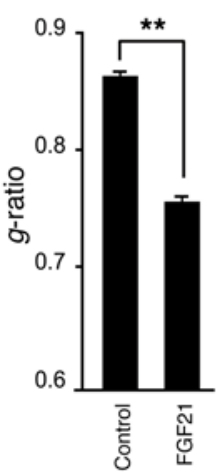


Figure 5. $\beta$-Klotho expression in OPCs is required for remyelination. (A) Representative images of $\beta$-klotho expression in the mouse spinal cord 3 days after LPC injection. (B) Representative images of spinal cord sections double-labeled for PDGFR $\alpha$ and Ki67. Graph shows quantitations as indicated in images ( $n=3$ for control, 3 for CKO); ${ }^{*} P<0.05$. Arrows indicate Ki67+ cells colabeled with PDGFR $\alpha$. (C) Representative images of spinal cord sections labeled for MBP. Graph shows quantitations as indicated in the images $\left(n=4\right.$ each); ${ }^{* *} P<0.01$. (D) Representative images of brain sections double-labeled for PDGFR $\alpha$ and Ki67 in the mouse cortex, 7 days after traumatic brain injury. FGF21 was administered i.c.v. 24 hours after LPC injection $(n=4) ;{ }^{*} P<0.05$. Arrows indicate Ki67 ${ }^{+}$cells colabeled with PDGFR $\alpha$; arrowheads indicate Ki67 ${ }^{+}$cells not labeled with PDCFR $\alpha$. (E) Representative images of brain sections labeled for MBP in the mouse cortex, 14 days after traumatic brain injury. Graph shows quantitations as indicated in the images ( $n=6$ for vehicle, $n=4$ for FGF21); ${ }^{*} P<0.05$. (F) Representative immunoelectron microscopy images of myelin in the mouse cortex, 14 days after traumatic brain injury. Graphs show quantitations of $g$-ratio indicated in the images $(n=4) ;{ }^{*} P<0.01$ as determined by Student's $t$ test. Error bars represent SEM. Scale bars: $20 \mu \mathrm{m}$ (A); $50 \mu \mathrm{m}$ (B and D); $200 \mu \mathrm{m}$ (C and E); $2 \mu \mathrm{m}$ (F).

Vascular barrier permeability assays. Mice were transcardially perfused with $4 \%$ paraformaldehyde (PFA) in PBS. Spinal cord tissues were immersed in $30 \%$ sucrose in PBS overnight at $4^{\circ} \mathrm{C}$. Cryosections (30- $\mu \mathrm{m}$ thickness) were mounted on Matsunami adhesive silanecoated slides (Matsunami Glass) and permeabilized in PBS containing $0.05 \%$ Tween-20 and $2 \%$ BSA for 1 hour at room temperature. To detect IgG extravasation, sections were incubated with Alexa Fluor 568-conjugated goat anti-mouse IgG (1:500; Invitrogen, A-11004) for 1 hour at room temperature. In another set of experiments, mice were injected i.v. with $0.1 \mathrm{ml}$ of Alexa Fluor 488-conjugated cadaverine at a concentration of $1 \mathrm{mg} / \mathrm{ml}$ (Life Technologies, A30676) 2 hours before fixation. Spinal cord tissues were immersed in 30\% sucrose in PBS for 24 hours at $4^{\circ} \mathrm{C}$, after which they were sectioned at 30- $\mu$ m thickness on a cryostat and mounted on Matsunami adhesive silane-coated slides. All samples were analyzed by laser scanning confocal microscopy (Olympus FV1200).

Evans blue leakage. Evans blue dissolved at $5 \mathrm{mg} / \mathrm{ml}$ in saline was injected into the tail vein of mice at a dose of $10 \mathrm{mg} / \mathrm{kg} 24$ hours before the end of breeding. Mice were intracardially perfused with PBS, and thoracic spinal cord at the level of Th11-12 was dissected out following immersion for 48 hours at $4^{\circ} \mathrm{C}$ in $200 \mu \mathrm{l}$ of formamide. The supernatants from the samples were collected, and the concentration of Evans blue in the supernatants was determined spectrophotometrically at $620 \mathrm{~nm}$.

Immunohistochemistry. At 0, 3, 7, or 14 days after LPC injection, mice were transcardially perfused with PBS followed by $4 \%$ PFA in $0.1 \mathrm{M}$ phosphate buffer (PB). Brain, spinal cord, and pancreas were removed and were postfixed with $4 \%$ PFA in PB for 1 hour (for PDGFR $\alpha$ staining) or overnight at $4^{\circ} \mathrm{C}$ following immersion in $30 \%$ sucrose in PBS. Tissues were embedded in OCT compound (TissueTek), and then $30-\mu \mathrm{m}$ sections were cut and mounted on Matsunami adhesive silane-coated slides (Matsunami Glass). To detect BrdU-labeled cells, the sections were pretreated with $2 \mathrm{~N} \mathrm{HCl}$ for 30 minutes at $37^{\circ} \mathrm{C}$ and washed in $0.1 \mathrm{M}$ borate buffer ( $\mathrm{pH}$ 8.5) for 10 minutes. The sections were permeabilized with PBS containing $0.3 \%$ Triton X-100 and 10\% Goat Serum (Sigma-Aldrich) (for PDGFR $\alpha$ staining) or PBS containing 0.1\% Triton X-100 and 5\% BSA for 1 hour at room temperature. The sections were then incubated with primary antibodies overnight at $4^{\circ} \mathrm{C}$, and then incubated with fluorescently labeled secondary antibody for 1 hour at room temperature. The primary antibodies used were as follows: rat anti-mouse PDGFR $\alpha$ (1:400; 558774, BD Biosciences), rabbit anti-human Ki67 (1:200; ab16667, Abcam), goat anti-human MBP (1:500; sc-13914, Santa Cruz Biotechnology), mouse anti-BrdU (1:100; M0744, Dako), mouse anti-bovine O4 (1:250; MAB345, Millipore), rabbit antihuman Adenomatous polyposis coli (APC) (1:250; sc-896, Santa Cruz Biotechnology), rabbit anti-human $\beta$-klotho (1:300; HPA021136, Sigma-Aldrich), rabbit anti-human FGF21 (1:200; ab64857, Abcam), mouse anti-porcine glucagon (1:500; G2654, SigmaAldrich), mouse anti-human insulin (1:500; ab6995, Abcam), and rat anti-human somatostatin (1:200; MAB354, Millipore). Alexa Fluor 488- or 568-conjugated goat antibody against rabbit IgG, goat antibody against rat IgG, goat antibody against mouse IgG, goat antibody against mouse IgM, and donkey antibody against goat IgG were used as secondary antibodies. In another set of experiments, we also used Alexa Fluor 647-conjugated mouse anti-human Ki67 antibody (1:100; 558615, BD Biosciences) and Alexa Fluor 488conjugated anti-NeuN antibody (1:100; MAB377X, Millipore). We validated all antibodies for use in this study. To estimate the numbers of proliferating and total OPCs, the sections were immunostained with PDGFR $\alpha$-specific and Ki67-specific antibodies. The numbers of PDGFR $\alpha^{+} \mathrm{Ki} 67^{+}$cells were counted in sections around the LPC lesion (segments Th11-12, just caudal to the lesion center). The means were calculated from 3 sections spaced $100 \mu \mathrm{m}$ apart. All samples were analyzed by laser scanning confocal microscopy (Olympus FV1200).

Serum preparation from adult mice. Cardiac blood was collected from adult C57BL/6J mice and allowed to clot for 30 minutes at room temperature. After centrifugation at $1,000 \mathrm{~g}$ for 20 minutes, the supernatant was collected and stored at $-80^{\circ} \mathrm{C}$. For digestion, serum was incubated at $37^{\circ} \mathrm{C}$ for 2 hours with DNase (10 $\mu \mathrm{g} / \mathrm{ml}$; Sigma-Aldrich), RNase $(100 \mu \mathrm{g} / \mathrm{ml}$; Roche), or Pronase (3 U/ml; Roche). For heat denaturation, serum was heated at $95^{\circ} \mathrm{C}$ for 10 minutes.

Primary culture of OPCs and cell proliferation assay. Primary cultures of OPCs were obtained from C57BL/6J mice at postnatal day 1. Whole brains were dissected in HBSS (Life Technologies) and dissociated into single-cell suspensions using the Neural Tissue Dissociation Kit (Miltenyi Biotec). OPCs were isolated by A2B5- and PDGFR $\alpha$ specific antibody-coated magnetic beads (Miltenyi Biotec). The cells were plated on poly-L-lysine-precoated (PLL-precoated) 96-well plates (Greiner Bio-One) at a density of $1 \times 10^{4}$ cells per well. The cells were maintained at $37^{\circ} \mathrm{C}$ with $5 \% \mathrm{CO}_{2}$ and cultured in DMEM containing $1 \mathrm{mM}$ sodium pyruvate (Sigma-Aldrich), 0.1\% BSA (Sigma-Aldrich), $50 \mu \mathrm{g} / \mathrm{ml}$ apo-transferrin (Sigma-Aldrich), $5 \mu \mathrm{g} / \mathrm{ml}$ insulin (SigmaAldrich), $30 \mathrm{nM}$ sodium selenite (Sigma-Aldrich), $10 \mathrm{nM}$ biotin (SigmaAldrich), $10 \mathrm{nM}$ hydrocortisone (Sigma-Aldrich), $10 \mathrm{ng} / \mathrm{ml}$ PDGF-AA (PeproTech), $10 \mathrm{ng} / \mathrm{ml}$ basic FGF (PeproTech), and 1\% penicillin/ streptomycin (Life Technologies). The culture conditions we used were suitable for OPC culture, as previously described (65). Immunocytochemistry revealed that $93.4 \% \pm 0.01 \%$ of cells in $\mathrm{A} 2 \mathrm{~B} 5^{+}$cell culture were labeled with PDGFR $\alpha$, an OPC marker (data not shown).

To conduct pharmacological screening, we used the Inhibitor Select 384-well Protein Kinase Inhibitor Library I (Calbiochem). Cells were pretreated with various compounds in the library for 30 minutes, and then cultured in the presence of mouse serum. To inhibit FGFR signaling, cells were pretreated with PD173074 (Sigma-Aldrich) and 
A
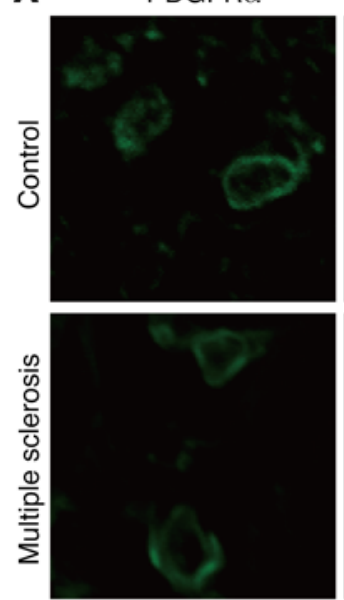

$\beta$-Klotho

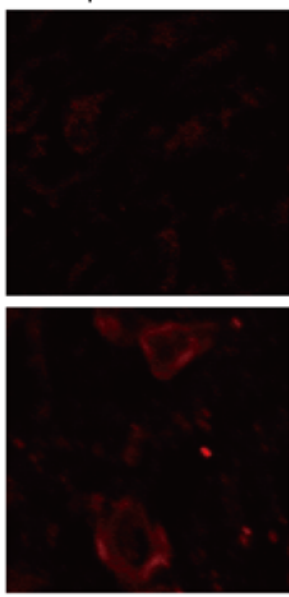

Overlay

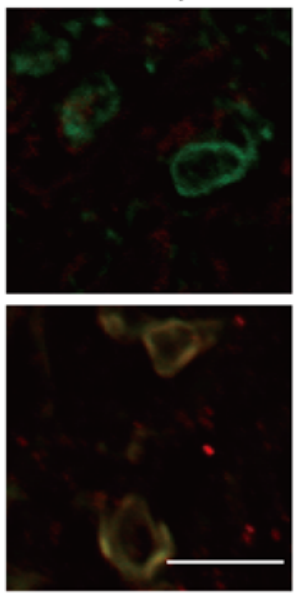

B

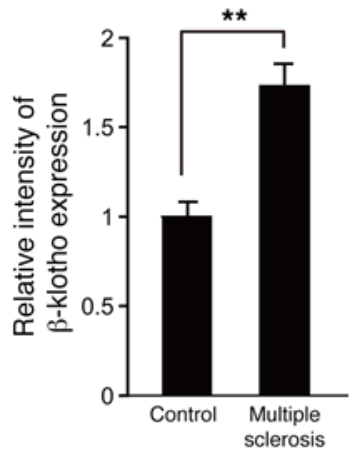

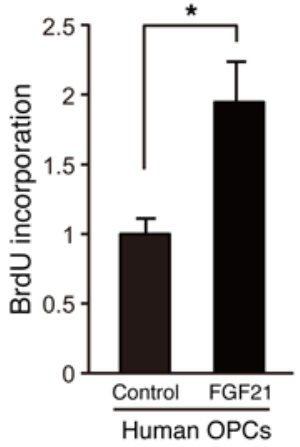

Figure 6. FGF21 promotes human OPC proliferation. (A) Representative image of $\beta$-klotho expression in an autopsied sample from healthy patient and a patient with multiple sclerosis. Graphs show quantitations as indicated in the images ( $n=4$ for healthy patients, 3 for multiple sclerosis patients); ${ }^{* *} P<$ 0.01. (B) BrdU incorporation in human OPCs after stimulation with recombinant FGF21 ( $n=6$ for control, 4 for FGF21); ${ }^{*} P<0.05$ as determined by Student's $t$ test. Error bars represent SEM. Scale bar: $20 \mu \mathrm{m}$.

NF449 (Calbiochem) for 30 minutes, and then incubated in the presence of mouse serum. To assess the role of FGFs, we used recombinant mouse FGF15 (Abcam), recombinant mouse FGF21 (R\&D Systems), and recombinant mouse FGF23 (R\&D Systems). For neutralization experiments, neutralizing antibodies against mouse FGF21 (final concentration, $6 \mu \mathrm{g} / \mathrm{ml}$; sc-16842, Santa Cruz Biotechnology) were added to the culture 30 minutes before serum treatment. Control samples were treated with normal goat IgG (Sigma-Aldrich).

To assess cell proliferation, cells were incubated in DMEM supplemented with or without $1 \%$ (vol/vol) serum obtained from adult mice. BrdU was added into the culture 24 hours after the beginning of culture, and cell proliferation was estimated 48 hours after stimulation by measurement of BrdU incorporation into newly synthesized cellular DNA using the Cell Proliferation ELISA and BrdU (colorimetric) kit (Roche). Absorbances were measured by microplate reader (Molecular Devices SpectraMax 5).

BrdU detection by immunocytochemistry. OPCs were cultured in medium containing BrdU (10 $\mu \mathrm{M}$; Sigma-Aldrich) for 2 hours. After culture, cells were fixed with 4\% PFA in PBS for 30 minutes at room temperature, and then cells were pretreated with $2 \mathrm{~N} \mathrm{HCl}$ for $30 \mathrm{~min}$ utes at $37^{\circ} \mathrm{C}$ and washed in $0.1 \mathrm{M}$ borate buffer $(\mathrm{pH} 8.5)$ for 10 minutes. After permeabilization with PBS containing 0.3\% Triton X-100 and $10 \%$ goat serum (Sigma-Aldrich), cells were incubated with primary antibodies overnight at $4^{\circ} \mathrm{C}$. Cells were incubated with fluorescent dye-conjugated secondary antibody for 1 hour at room temperature. The primary antibodies used were as follows: rat anti-mouse PDGFR $\alpha$ (1:500; 558774, BD Biosciences), goat anti-human MBP (1:500; sc-13914, Santa Cruz Biotechnology), mouse anti-pig GFAP (1:400; G3893, Sigma-Aldrich), and mouse anti-BrdU (1:100; M0744, Dako). The secondary antibodies used were as follows: Alexa Fluor 488or 568-conjugated goat antibody against rabbit IgG, goat antibody against rat IgG, and goat antibody against mouse IgG.

Preparation and transfection of siRNA. Mouse FGF21 siRNA and $\beta$-klotho siRNA were synthesized by Ambion (Life Technologies). Mouse FGFR siRNAs were synthesized by Thermo Fisher Scientific. The sense and antisense strands of siRNA were as follows: FGF21 siRNA, 5'-GCCAGAUGGAGCUCUCUAUTT-3' (sense) and 5'-AUAGAGAGCUCCAUCUGGCTG-3' (antisense); $\beta$-klotho siRNA, 5'-GGAUUAAACUGGAAUACGATT-3' (sense) and 5'-UCGUAUUCCAGUUUAAUCCAG-3' (antisense); FGFR1 siRNA, 5'-GCAUCGUGGAGAAUGAGUAtt-3' (sense) and 5'-UACUCAUUCUCCACGAUGCag-3' (antisense); FGFR2 siRNA, 5'-CUCUCUACGUCAUAGUUGAtt-3' (sense) and 5'-CUCUCUACGUCAUAGUUGAtt-3' (antisense); FGFR3 siRNA, 5'-GAGUGAUGUUUGGUCUUUUtt-3' (sense) and 5'-AAAAGACCAAACAUCACUCtg-3' (antisense); FGFR4 siRNA, 5'-CAUUGACUACUAUAAGAAAtt-3' (sense) and 5'-UUUCUUAUAGUAGUCAAUGtg-3' (antisense). Transfection of cultured OPCs with siRNAs targeting FGFRs and $\beta$-klotho was performed using Lipofectamine RNAiMAX (Invitrogen). The cells were lysed after 48 hours, and the lysates were subjected to real-time PCR.

For knockdown of FGF21 in pancreas, FGF21 siRNAs were mixed with in vivo-jetPEI reagent (Polyplus Transfection). Mice received intrapancreatic injection of either PEI-conjugated FGF21 siRNA or PEI-conjugated nontargeting siRNA $(0.02 \mu \mathrm{g} /$ mouse, 3 points of a pancreas) as previously described with slight modifications (36) just after LPC injection. The tissue was lysed after 48 hours, and the lysates were subjected to real-time PCR. Injection of siRNA into the pancreas did not result in any signs of toxicity at the behavioral level (data not shown).

Quantitative reverse transcriptase PCR. Total RNA was isolated using the RNeasy Mini kit (Qiagen), and cDNA was synthesized using the High Capacity cDNA Reverse Transcriptase Kit (Applied Biosyste$\mathrm{ms}$ ). cDNA fragments were amplified using the following primer pairs: FGF21 forward, GCTGCTGGAGGACGGTTACA; FGF21 reverse, CACAGGTCCCCAGGATGTTG; FGFR1 forward, CGCTCTACCTGGAGATCATT; FGFR1 reverse, ATAAAGAGGACCATCCTGTG; FGFR2 forward, CACCAACTGCACCAATGAAC; FGFR2 reverse, GGCTGGGTGAGATCCAAGTA; FGFR3 forward, CATCCGGCAGACATACACAC; FGFR3 reverse, TTCACTTCCACGTGCTTCAG; FGFR4 forward, CGCCAGCCTGTCACTATACAAA; FGFR4 reverse, CCAGAGGACCTCGACTCCAA; $\beta$-klotho forward, TCCCCTGTGATTTCTCTTGG; $\beta$-klotho reverse, GAGCAATCTGTTGCCAGTGA; 
GAPDH forward, TCACCACCATGGAGAAGGC; GAPDH reverse, GCTAAGCAGTTGGTGGTGCA. Samples for SYBR Green assays consisted of a $1 \times$ final concentration of Power SYBR Green PCR Master Mix (Applied Biosystems), $200 \mathrm{nM}$ gene-specific primers, and $10 \mathrm{ng}$ cDNA (ABI ViiA7 real-time PCR system; Applied Biosystems). PCR conditions included 1 cycle at $95^{\circ} \mathrm{C}$ for 10 minutes, followed by 40 cycles of $95^{\circ} \mathrm{C}$ for 15 seconds and $60^{\circ} \mathrm{C}$ for 60 seconds. A melting analysis was carried out following PCR to monitor amplification specificity. Relative mRNA expression was normalized against GAPDH mRNA levels in the same samples, and calculated by the $\Delta / \Delta$ Ct method.

MTT assay. PDGFR $\alpha^{+}$OPCs were plated on PLL-coated 96-well plates at a density of $1 \times 10^{4}$ cells per well. Cells were transfected with $\beta$-klotho siRNA using Lipofectamine RNAiMAX. After 72 hours of culture, MTT solution was added to each well, and the cultures were incubated for an additional 4 hours. Crystalline formazan was solubilized with $100 \mu \mathrm{l}$ of a $10 \%$ (wt/vol) SDS solution for 24 hours. Absorbance at $595 \mathrm{~nm}$ was read spectrophotometrically using a microplate reader.

Differentiation assay. Primary cultures of OPCs were obtained from $\mathrm{C} 57 \mathrm{BL} / 6 \mathrm{~J}$ mice at postnatal day 1 . Whole brains were dissected in PBS and dissociated into single-cell suspensions by incubation at $37^{\circ} \mathrm{C}$ for 15 minutes in $0.25 \%$ trypsin-PBS. After neutralization by DMEM containing $10 \%$ FBS, cells were centrifuged at $300 \mathrm{~g}$ for $5 \mathrm{~min}$ utes, suspended in 10\% FBS-DMEM, and filtered through a $100-\mu \mathrm{m}$ nylon cell strainer. Single cells were plated on PLL-precoated $10-\mathrm{cm}$ dishes (Greiner Bio-One) and maintained at $37^{\circ} \mathrm{C}$ with $7 \% \mathrm{CO}_{2}$ in $10 \%$ FBS-DMEM. After 10 days of culture, cells were detached by treatment with $0.05 \%$ trypsin-PBS at $35^{\circ} \mathrm{C}$ for 4 minutes. Collected cells were centrifuged at $300 \mathrm{~g}$ for 5 minutes, and the pellets were suspended in OPC medium (DMEM containing $1 \mathrm{mM}$ sodium pyruvate [Sigma-Aldrich], 0.1\% BSA [Sigma-Aldrich], $50 \mu \mathrm{g} / \mathrm{ml}$ apo-transferrin [Sigma-Aldrich], $5 \mu \mathrm{g} / \mathrm{ml}$ insulin [Sigma-Aldrich], $30 \mathrm{nM}$ sodium selenite [Sigma-Aldrich], $10 \mathrm{nM}$ biotin [Sigma-Aldrich], $10 \mathrm{nM}$ hydrocortisone [Sigma-Aldrich], $10 \mathrm{ng} / \mathrm{ml}$ PDGF-AA [PeproTech], $10 \mathrm{ng} / \mathrm{ml}$ basic FGF [PeproTech], and 1\% penicillin/streptomycin [Life Technologies]). Cells were plated on noncoated dishes for 1 hour to separate cell types based on differences in adhesion. Cells that did not adhere after incubation (OPCs) were collected and plated on PLL-precoated 96-well plates at a density of $1 \times 10^{4}$ cells per well, and then maintained at $37^{\circ} \mathrm{C}$ with $5 \% \mathrm{CO}_{2}$ in OPC medium.

To induce oligodendrocyte differentiation, cells were cultured for an additional 3 days in DMEM containing $1 \mathrm{mM}$ sodium pyruvate (Sigma-Aldrich), 0.1\% BSA (Sigma-Aldrich), $50 \mu \mathrm{g} / \mathrm{ml}$ apo-transferrin (Sigma-Aldrich), $5 \mu \mathrm{g} / \mathrm{ml}$ insulin (Sigma-Aldrich), $30 \mathrm{nM}$ sodium selenite (Sigma-Aldrich), $10 \mathrm{nM}$ biotin (Sigma-Aldrich), $10 \mathrm{nM}$ hydrocortisone (Sigma-Aldrich), $30 \mathrm{ng} / \mathrm{ml}$ Triiodo-L-Thyronine (T3; SigmaAldrich), and $1 \%$ penicillin/streptomycin (Life Technologies) with or without recombinant mouse FGF21 (R\&D Systems).

Primary culture of astrocytes. Primary cultures of OPCs were obtained from C57BL/6J mice at postnatal day 1. Whole brains were dissected in PBS and dissociated into single-cell suspensions by incubation at $37^{\circ} \mathrm{C}$ for 15 minutes in $0.25 \%$ trypsin-PBS. After neutralization by $10 \%$ FBS-DMEM, cells were centrifuged at $300 \mathrm{~g}$ for 5 minutes, suspended in 10\% FBS-DMEM, and filtered through a 100- $\mu$ m nylon cell strainer. Single cells were plated on PLL-coated 10-cm dishes and maintained at $37^{\circ} \mathrm{C}$ with $5 \% \mathrm{CO}_{2}$ in $10 \%$ FBS-DMEM. Ten days after culture, cells were detached by treatment with $0.25 \%$ trypsin-PBS and neutralized with an equal volume of 10\% FBS-DMEM. The cells were pelleted by centrifugation at $1,000 \mathrm{~g}$ for 3 minutes, and then resuspended in $10 \%$ FBS-DMEM. The cells were plated on culture plates precoated with PLL. After 2 passages, the cells were plated on PLL-coated 96-well plates at a density of $1 \times 10^{4}$ cells per well. To collect astrocyte supernatant, the cells were cultured in DMEM with or without $1 \%$ (vol/ vol) serum obtained from adult mice. One day after incubation, supernatant was collected and added to the OPC culture.

ELISA. FGF21 levels in mouse serum or tissue lysates were examined using a Mouse/Rat FGF21 Quantikine ELISA (R\&D Systems). The tissues were homogenized in the lysis buffer (25 mM Tris- $\mathrm{HCl}$ [pH 7.5], $150 \mathrm{mM} \mathrm{NaCl}, 1 \%$ Triton X-100, and $1 \mathrm{mM}$ EDTA containing protease inhibitor [Roche]). The lysates were clarified by centrifugation at 13,000 $g$ at $4^{\circ} \mathrm{C}$ for 20 minutes, and the supernatants were used for assay.

FGF21 labeling. Recombinant mouse FGF21 was conjugated with HiLyte Fluor 555 labeling kit (Dojindo Molecular Technologies). To detect leakage of FGF21 into the spinal cord, mice received fluorescencelabeled FGF21 (200 pg/mouse) i.v. 2 hours before sampling. Spinal cord tissues were isolated after PBS perfusion and were homogenized in lysis buffer. After centrifugation (13,000 $g$ at $4^{\circ} \mathrm{C}$ for 20 minutes), fluorescence in the supernatant was measured by microplate reader.

BrdU labeling in vivo. BrdU $(1 \mathrm{mg} / \mathrm{ml})$ was added to the drinking water (supplemented with $1 \%$ [wt/vol] sucrose) for 5 weeks before sacrifice of the mice. The water bottles containing BrdU were protected from light and changed every 3 days during labeling periods.

Electron microscopy. Mice were transcardially perfused with $0.1 \mathrm{M}$ $\mathrm{PB}$ containing $2.5 \%$ glutaraldehyde and $2 \%$ PFA. Spinal cord tissues were removed and were postfixed in the same fixative at $4^{\circ} \mathrm{C}$ overnight. The spinal cords were sliced into $50-\mu \mathrm{m}$ pieces using a Vibrating Blade Microtome (VT1000s, Leica) and washed in 0.1 M PB to prepare the sample for electron microscopy as described previously (66). The samples were incubated in $0.1 \mathrm{M} \mathrm{PB}$ containing $1 \% \mathrm{OsO}_{4}$ (TAAB) in 0.1 $\mathrm{M}$ PB for 1 hour and dehydrated in an ascending ethanol series, and then embedded in epoxy resins through propylene oxide (Wako). The samples were cut into 80-nm sections using a Reichert-type ultramicrotome (Ultracut N, Leica), and ultrathin sections on formvar-coated 1-hole $\mathrm{Cu}$ grids (Nisshin EM Corp.) were observed by transmission electron microscopy (H-7650, Hitachi). Nonoverlapping obtained images of nerve fiber cross sections were analyzed using Image J version $1.44 \mathrm{p}(\mathrm{NIH})$. The diameter of axons, myelin thickness, and $g$-ratio were determined from about 600 fibers in the corticospinal tract.

Ladder-walk test. We observed the walking of mice on a horizontal ladder (whole length of $1 \mathrm{~m}$ ) with stainless steel rungs spaced 1-4 $\mathrm{cm}$ apart. Mice were habituated to the apparatus before the surgery. The number of faulty placements of the injured hind paw was counted when the mice walked through the 1-m stretch. Deep slips/misses, minor slips, and placement errors (correction, replacement) were considered as faults (67). The tests were performed during the light cycle. The control baseline scores were obtained just before the injury. The spacing between rungs was changed accordingly to prevent the animal from learning the rung locations.

IHC staining of human tissues and cell proliferation assay of human OPCs. This study was approved by the Local Ethical Committee, Toneyama National Hospital (Toyonaka, Japan), with research number 1135-1 (Research Resource Network, Japan), and by the Local Ethical Committee, Aichi Medical University (Nagakute, Japan), with research number 15-017 (Aichi Medical University, Kareiken Brain Resource Center). Informed consent was obtained at the occasion 
of autopsy (brain banking). We obtained autopsy brain tissues from 4 control individuals ( 3 men; median age: 68 years; range: $63-70$ years) and 3 individuals with multiple sclerosis (1 man; median age: 66 years; range: $63-85$ years). Informed consents were obtained from family member of patients. Formalin-fixed spinal cord samples were embedded in paraffin and cut into $10-\mu \mathrm{m}$-thick sections for IHC. Sections were deparaffinized and were permeabilized with PBS containing $0.3 \%$ Triton X-100 and 5\% BSA for 1 hour at room temperature. Sections were incubated with primary antibodies against mouse antihuman PDGFR $\alpha$ (1:50; 0100-0220, Serotec) and rabbit anti-human $\beta$-klotho (1:50; AV53325, Sigma-Aldrich) and Alexa Fluor 488-conjugated goat anti-mouse IgG and/or Alexa Fluor 647-conjugated goat anti-rabbit IgG (Invitrogen) secondary antibodies. The sections were treated with a solution of Sudan Black B. All images were captured by laser scanning confocal microscopy. The research protocol was approved by the Human Use Review Committees of the Graduate School of Medicine, Osaka University. Informed consent was obtained from all subjects.

Primary cultures of human OPCs were obtained from ScienCell. The cells were plated on PLL-precoated 96-well plates at a density of $1 \times 10^{4}$ cells per well, and then maintained at $37^{\circ} \mathrm{C}$ with $5 \% \mathrm{CO}_{2}$ in DMEM containing $1 \mathrm{mM}$ sodium pyruvate (Sigma-Aldrich), 0.1\% BSA (Sigma-Aldrich), $50 \mu \mathrm{g} / \mathrm{ml}$ apo-transferrin (Sigma-Aldrich), $5 \mu \mathrm{g} / \mathrm{ml}$ insulin (Sigma-Aldrich), $30 \mathrm{nM}$ sodium selenite (Sigma-Aldrich), 10 $\mathrm{nM}$ biotin (Sigma-Aldrich), $10 \mathrm{nM}$ hydrocortisone (Sigma-Aldrich), 10 ng/ml PDGF-AA (PeproTech), $10 \mathrm{ng} / \mathrm{ml}$ basic FGF (PeproTech), and $1 \%$ penicillin/streptomycin (Life Technologies). To assess cell proliferation, cells were cultured in DMEM supplemented with or without recombinant human FGF21 (R\&D Systems) at a final concentration of $6 \mu \mathrm{g} / \mathrm{ml}$. BrdU was added to the culture 24 hours after the beginning of culture, and cell proliferation was estimated 48 hours after stimulation by measurement of BrdU incorporation into newly synthesized cellular DNA using the Cell Proliferation ELISA and BrdU (colorimetric) kit.

Statistics. Data are presented as means \pm SEM. Statistical significance between groups was determined by unpaired Student's 2-tailed $t$ test, paired $t$ test, or repeated-measures ANOVA followed by post hoc comparison with Tukey-Kramer test or Dunnett's test. $P$ less than 0.05 was considered to represent a significant difference. Data distribution was assumed to be normal. No randomization was used to assign experimental groups or collect data, but samples were assigned to specific experimental groups without bias. No data points were excluded. Histopathological analyses and behavioral tests were performed in a blinded manner.

\section{Author contributions}

M Kuroda performed almost all experiments and analyzed the data. RM developed the concept, designed the experiments, and wrote the manuscript. NM performed immunohistochemical experiments. YK carried out electron microscopy analyses. $\mathrm{MH}$ helped with tamoxifen injection and cuprizone feeding. HF, MY, and HM provided autopsied samples. M Konishi and NI contributed to FGF21-KO mouse experiments. TY supervised all aspects of this project.

\section{Acknowledgments}

We are grateful to Seiji Takashima and Akira Kikuchi for technical advice and Ayumu Shintani for statistical analysis. We also thank Steven Kliewer and Yuan Zhang for providing $\beta$-klotho-floxed mice. This work was supported by a Grant-in-Aid from Precursory Research for Embryonic Science and Technology to RM, a Grant-inAid for Scientific Research (B) from the Japan Society for the Promotion of Science (16H04672) to RM, and a Grant-in-Aid for Scientific Research (S) from the Japan Society for the Promotion of Science (25221309) to TY. We also thank the Osaka University Program for the Support of Networking among Present and Future Researchers and the Center of Medical Research and Education, Graduate School of Medicine, Osaka University for technical support.

Address correspondence to: Rieko Muramatsu or Toshihide Yamashita, Department of Molecular Neuroscience, Graduate School of Medicine, Osaka University, 2-2 Yamadaoka, Suita, Osaka 565-0871, Japan. Phone: 81.6.68793663; E-mail: muramatsu@ molneu.med.osaka-u.ac.jp (R. Muramatsu); yamashita@molneu. med.osaka-u.ac.jp (T. Yamashita).
1. Chiaravalloti ND, DeLuca J. Cognitive impairment in multiple sclerosis. Lancet Neurol. 2008;7(12):1139-1151.

2. Harel NY, Strittmatter SM. Can regenerating axons recapitulate developmental guidance during recovery from spinal cord injury? Nat Rev Neurosci. 2006;7(8):603-616.

3. Franklin RJ, Ffrench-Constant C. Remyelination in the CNS: from biology to therapy. Nat Rev Neurosci. 2008;9(11):839-855.

4. Scolding N, Franklin R, Stevens S, Heldin CH, Compston A, Newcombe J. Oligodendrocyte progenitors are present in the normal adult human CNS and in the lesions of multiple sclerosis. Brain. 1998;121(pt 12):2221-2228.

5. Dawson MR, Polito A, Levine JM, Reynolds R. NG2-expressing glial progenitor cells: an abundant and widespread population of cycling cells in the adult rat CNS. Mol Cell Neurosci. 2003;24(2):476-488.

6 . Yeung MS, et al. Dynamics of oligodendrocyte generation and myelination in the human brain.
Cell. 2014;159(4):766-774.

7. Duncan ID, Brower A, Kondo Y, Curlee JF, Schultz RD. Extensive remyelination of the CNS leads to functional recovery. Proc Natl Acad Sci US A. 2009;106(16):6832-6836.

8. Edgar JM, Nave KA. The role of CNS glia in preserving axon function. Curr Opin Neurobiol. 2009;19(5):498-504.

9. Smith KJ, Blakemore WF, McDonald WI. Central remyelination restores secure conduction. Nature. 1979;280(5721):395-396.

10. Moore CS, Abdullah SL, Brown A, Arulpragasam A, Crocker SJ. How factors secreted from astrocytes impact myelin repair. J Neurosci Res. 2011;89(1):13-21.

11. Clemente D, Ortega MC, Melero-Jerez C, de Castro F. The effect of glia-glia interactions on oligodendrocyte precursor cell biology during development and in demyelinating diseases. Front Cell Neurosci. 2013;7:268.

12. Baron W, Metz B, Bansal R, Hoekstra D, de Vries H. PDGF and FGF-2 signaling in oligodendrocyte progenitor cells: regulation of proliferation and differentiation by multiple intracellular signaling pathways. Mol Cell Neurosci. 2000;15(3):314-329.

13. Arai K, Lo EH. An oligovascular niche: cerebral endothelial cells promote the survival and proliferation of oligodendrocyte precursor cells. JNeurosci. 2009;29(14):4351-4355.

14. Takahashi C, Muramatsu R, Fujimura H, Mochizuki H, Yamashita T. Prostacyclin promotes oligodendrocyte precursor recruitment and remyelination after spinal cord demyelination. Cell Death Dis. 2013;4:e795.

15. Persidsky Y, Ramirez SH, Haorah J, Kanmogne GD. Blood-brain barrier: structural components and function under physiologic and pathologic conditions. J Neuroimmune Pharmacol. 2006;1(3):223-236.

16. Zlokovic BV. The blood-brain barrier in health and chronic neurodegenerative disorders. Neuron. 2008;57(2):178-201.

17. Ruckh JM, et al. Rejuvenation of regeneration in the aging central nervous system. Cell Stem Cell. 
2012;10(1):96-103.

18. Muramatsu R, et al. Prostacyclin prevents pericyte loss and demyelination induced by lysophosphatidylcholine in the central nervous system. J Biol Chem. 2015;290(18):11515-11525.

19. Le Menuet $D$, Lombès $M$. The neuronal mineralocorticoid receptor: from cell survival to neurogenesis. Steroids. 2014;91:11-19.

20. Ziegler AN, Levison SW, Wood TL. Insulin and IGF receptor signalling in neural-stem-cell homeostasis. Nat Rev Endocrinol. 2015;11(3):161-170.

21. Kurosu H, et al. Tissue-specific expression of betaKlotho and fibroblast growth factor (FGF) receptor isoforms determines metabolic activity of FGF19 and FGF21. J Biol Chem. 2007;282(37):26687-26695.

22. Kharitonenkov A, et al. FGF-21/FGF-21 receptor interaction and activation is determined by betaKlotho. J Cell Physiol. 2008;215(1):1-7.

23. Suzuki M, et al. betaKlotho is required for fibroblast growth factor (FGF) 21 signaling through FGF receptor (FGFR) 1c and FGFR3c. Mol Endocrinol. 2008;22(4):1006-1014.

24. Adams AC, Cheng CC, Coskun T, Kharitonenkov A. FGF21 requires $\beta$ klotho to act in vivo. PLoS One. 2012;7(11):e49977.

25. Bai L, et al. Hepatocyte growth factor mediates mesenchymal stem cell-induced recovery in multiple sclerosis models. Nat Neurosci. 2012;15(6):862-870.

26. Aguirre A, Dupree JL, Mangin JM, Gallo V. A functional role for EGFR signaling in myelination and remyelination. Nat Neurosci. 2007;10(8):990-1002.

27. Deverman BE, Patterson PH. Exogenous leukemia inhibitory factor stimulates oligodendrocyte progenitor cell proliferation and enhances hippocampal remyelination. J Neurosci. 2012;32(6):2100-2109.

28. Kondo A, Nakano T, Suzuki K. Blood-brain barrier permeability to horseradish peroxidase in twitcher and cuprizone-intoxicated mice. Brain Res. 1987;425(1):186-190.

29. McMahon EJ, Suzuki K, Matsushima GK. Peripheral macrophage recruitment in cuprizone-induced CNS demyelination despite an intact blood-brain barrier. J Neuroimmunol. 2002;130(1-2):32-45.

30. St-Germain JR, et al. Multiple myeloma phosphotyrosine proteomic profile associated with FGFR3 expression, ligand activation, and drug inhibition. Proc Natl Acad Sci U S A. 2009;106(47):20127-20132.

31. Krejci P, et al. NF449 is a novel inhibitor of fibroblast growth factor receptor 3 (FGFR3) signaling active in chondrocytes and multiple myeloma cells. J Biol Chem. 2010;285(27):20644-20653.

32. Bookout AL, et al. FGF21 regulates metabolism and circadian behavior by acting on the nervous system. Nat Med. 2013;19(9):1147-1152.

33. Beenken A, Mohammadi M. The FGF family: biology, pathophysiology and therapy. Nat Rev Drug Discov. 2009;8(3):235-253.

34. Fon Tacer K, et al. Research resource: Compre- hensive expression atlas of the fibroblast growth factor system in adult mouse. Mol Endocrinol. 2010;24(10):2050-2064.

35. Hale C, et al. Lack of overt FGF21 resistance in two mouse models of obesity and insulin resistance. Endocrinology. 2012;153(1):69-80.

36. Leconet W, Petit P, Peraldi-Roux S, Bresson D. Nonviral delivery of small interfering RNA into pancreas-associated immune cells prevents autoimmune diabetes. Mol Ther. 2012;20(12):2315-2325

37. Bien-Ly N, et al. Lack of widespread BBB disruption in alzheimer's disease models: focus on therapeutic antibodies. Neuron. 2015;88(2):289-297.

38. Jeffery ND, Blakemore WF. Locomotor deficits induced by experimental spinal cord demyelination are abolished by spontaneous remyelination. Brain. 1997;120(pt 1):27-37.

39. Kinnunen KM, et al. White matter damage and cognitive impairment after traumatic brain injury. Brain. 2011;134(pt 2):449-463.

40. Shapira Y, Setton D, Artru AA, Shohami E. Bloodbrain barrier permeability, cerebral edema, and neurologic function after closed head injury in rats. Anesth Analg. 1993;77(1):141-148.

41. Baldwin SA, Fugaccia I, Brown DR, Brown LV, Scheff SW. Blood-brain barrier breach following cortical contusion in the rat. J Neurosurg. 1996;85(3):476-481.

42. Kharitonenkov A, et al. FGF-21 as a novel metabolic regulator. JClin Invest. 2005;115(6):1627-1635.

43. Hsuchou H, Pan W, Kastin AJ. The fasting polypeptide FGF21 can enter brain from blood. Peptides. 2007;28(12):2382-2386.

44. Tan BK, Hallschmid M, Adya R, Kern W, Lehnert H, Randeva HS. Fibroblast growth factor 21 (FGF21) in human cerebrospinal fluid: relationship with plasma FGF21 and body adiposity. Diabetes. 2011;60(11):2758-2762.

45. Owen BM, et al. FGF21 contributes to neuroendocrine control of female reproduction. Nat Med. 2013;19(9):1153-1156.

46. Zlokovic BV. Cerebrovascular permeability to peptides: manipulations of transport systems at the blood-brain barrier. Pharm Res. 1995;12(10):1395-1406.

47. Goetz R, Mohammadi M. Exploring mechanisms of FGF signalling through the lens of structural biology. Nat Rev Mol Cell Biol. 2013;14(3):166-180.

48. Furusho M, Roulois AJ, Franklin RJ, Bansal R. Fibroblast growth factor signaling in oligodendrocyte-lineage cells facilitates recovery of chronically demyelinated lesions but is redundant in acute lesions. Glia. 2015;63(10):1714-1728

49. Oh LY, et al. Fibroblast growth factor receptor 3 signaling regulates the onset of oligodendrocyte terminal differentiation. J Neurosci. 2003;23(3):883-894.

50. Redwine JM, Blinder KL, Armstrong RC. In situ expression of fibroblast growth factor receptors by oligodendrocyte progenitors and oligodendrocytes in adult mouse central nervous system. JNeurosci Res. 1997;50(2):229-237.

51. Redwine JM, Armstrong RC. In vivo prolifera- tion of oligodendrocyte progenitors expressing $\mathrm{PDGF} \alpha \mathrm{R}$ during early remyelination. J Neurobiol. 1998;37(3):413-428.

52. Fortin D, Rom E, Sun H, Yayon A, Bansal R. Distinct fibroblast growth factor (FGF)/FGF receptor signaling pairs initiate diverse cellular responses in the oligodendrocyte lineage. J Neurosci. 2005;25(32):7470-7479.

53. Messersmith DJ, Murtie JC, Le TQ, Frost EE, Armstrong RC. Fibroblast growth factor 2 (FGF2) and FGF receptor expression in an experimental demyelinating disease with extensive remyelination. J Neurosci Res. 2000;62(2):241-256.

54. Bansal R, Kumar M, Murray K, Morrison RS, Pfeiffer SE. Regulation of FGF receptors in the oligodendrocyte lineage. Mol Cell Neurosci. 1996;7(4):263-275.

55. Murakami M, et al. FGF-dependent regulation of VEGF receptor 2 expression in mice. JClin Invest. 2011;121(7):2668-2678.

56. Hayakawa K, et al. Vascular endothelial growth factor regulates the migration of oligodendrocyte precursor cells. J Neurosci. 2011;31(29):10666-10670.

57. Wittko-Schneider IM, Schneider FT, Plate KH. Brain homeostasis: VEGF receptor 1 and 2-two unequal brothers in mind. Cell Mol Life Sci. 2013;70(10):1705-1725.

58. Gaich G, et al. The effects of LY2405319, an FGF21 analog, in obese human subjects with type 2 diabetes. Cell Metab. 2013;18(3):333-340.

59. Li J, et al. Novel role of vitamin k in preventing oxidative injury to developing oligodendrocytes and neurons. J Neurosci. 2003;23(13):5816-5826.

60. Villeda SA, et al. The ageing systemic milieu negatively regulates neurogenesis and cognitive function. Nature. 2011;477(7362):90-94.

61. Katsimpardi L, et al. Vascular and neurogenic rejuvenation of the aging mouse brain by young systemic factors. Science. 2014;344(6184):630-634.

62. Villeda SA, et al. Young blood reverses age-related impairments in cognitive function and synaptic plasticity in mice. Nat Med. 2014;20(6):659-663.

63. Hotta $Y$, et al. Fibroblast growth factor 21 regulates lipolysis in white adipose tissue but is not required for ketogenesis and triglyceride clearance in liver. Endocrinology. 2009;150(10):4625-4633.

64. Miyake S, Muramatsu R, Hamaguchi M, Yamashita T. Prolyl hydroxylase regulates axonal rewiring and motor recovery after traumatic brain injury. Cell Death Dis. 2015;6:e1638.

65. Liu K, Tedeschi A, Park KK, He Z. Neuronal intrinsic mechanisms of axon regeneration. Annu Rev Neurosci. 2011;34:131-152.

66. Koyama Y, Nishida T, Tohyama M. Establishment of an optimised protocol for a Golgi-electron microscopy method based on a Golgi-Cox staining procedure with a commercial kit. J Neurosci Methods. 2013;218(1):103-109.

67. Metz GA, Whishaw IQ. Cortical and subcortical lesions impair skilled walking in the ladder rung walking test: a new task to evaluate fore- and hindlimb stepping, placing, and co-ordination. J Neurosci Methods. 2002;115(2):169-179. 